\title{
Multiple Chemical Sources Localization Using Virtual Physics-Based Robots with Release Strategy
}

\author{
Yuli Zhang, ${ }^{1,2}$ Xiaoping Ma, ${ }^{1}$ and Yanzi Miao' \\ ${ }^{1}$ School of Information and Electrical Engineering, China University of Mining and Technology, Xuzhou, Jiangsu 221116, China \\ ${ }^{2}$ School of Science, Dalian Jiaotong University, Dalian, Liaoning 116028, China \\ Correspondence should be addressed to Xiaoping Ma; xiaopingmacumt@126.com
}

Received 29 October 2014; Revised 5 January 2015; Accepted 8 January 2015

Academic Editor: Javier Moreno-Valenzuela

Copyright (c) 2015 Yuli Zhang et al. This is an open access article distributed under the Creative Commons Attribution License, which permits unrestricted use, distribution, and reproduction in any medium, provided the original work is properly cited.

\begin{abstract}
This paper presents a novel method of simultaneously locating chemical sources by a virtual physics-based multirobot system with a release strategy. The proposed release strategy includes setting forbidden area, releasing the robots from declared sources and escaping from it by a rotary force and goal force. This strategy can avoid the robots relocating the same source which has been located by other robots and leading them to move toward other sources. Various turbulent plume environments are simulated by Fluent and Gambit software, and a set of simulations are performed on different scenarios using a group of six robots or parallel search by multiple groups' robots to validate the proposed methodology. The experimental results show that release strategy can be successfully used to find multiple chemical sources, even when multiple plumes overlap. It can also extend the operation of many chemical source localization algorithms developed for single source localization.
\end{abstract}

\section{Introduction}

Chemical signals from any source propagate through a fluid environment in a peculiar way. They disperse through the environment by molecular diffusion and bulk flow [1]. The chemical source gradually dissolves into the ambient fluid medium resulting in a chemical trail or, in other words, an "odor plume." A plume is a dynamic structure composed of packets of odorant under the influence of fluid movements. A plume-tracing mobile robot is capable of tracing odor plumes and further locates a chemical source. The applications for employing autonomous robots to perform plume tracing and chemical source localization are widely ranging, for instance, searching for explosives and demining operations (by tracing volatile chemicals dispersed from the ordnance), judging toxic or harmful gas leakage location, checking for contraband (e.g., heroin), searching for survivors and casualties following a disaster, and antiterrorist attacks.

The chemical source localization task usually consists of three subtasks [2]: (1) finding the chemical plume; (2) tracing the chemical plume to the source; and (3) declaring the chemical source. There are three main characteristics of the problem of chemical source localization. First, there exists a global concentration maximum that occurs in the vicinity of the position of a chemical source and multiple local concentration maxima along the plume. Second, the positions with local concentration maxima are time varying due to the influence of wind. Finally, the chemical concentration is only detected within a plume at each time. On the basis of these three main characteristics, many chemical localization strategies have been proposed. According to the different possible contexts, existing methods can be divided into three categories. The first category is probabilistic strategies [3-5], which employ a spatial map keeping track of the probabilities for source presence. Such as "infotaxis" [5], the robot's movements can be determined such as to reduce the uncertainty on the source location. It is successful in the difficult context of turbulent conditions and a sensor suite consisting of a single chemical and wind sensor, but the computational complexity of the algorithms can be considerable. The second approach draws inspiration from biology. For example, chemical source localization algorithms have been based on the strategies of bacteria (Escherichia coli) [6], silkworm moths [7-9], dung beetles (Geotrupes stercorarius) [10], birds flocking [11, 12], 
and glowworms [13]. A drawback of biological strategies is that suitable behavior for the animals on which they are inspired may not be a suitable behavior for the robot. The third category is evolutionary approach [14]. This approach may be hard to formalize using mathematical approaches.

There have been many results on chemical source localization by using only one mobile robot $[15,16]$. Compared with a single robot, a multirobot system obviously provides several advantages, namely, wider detection region to capture the time-varying plume, faster exploration in a search environment, and system fault tolerance to individual robot failures. Cooperation is a key parameter in the performance of a group of robots which are trying to solve a general search problem [17]. The cooperation, communication, and management of the robots in a multiagent system can be done either in centralized way by using a base station as the server or decentralized way by having a distributed behavioral based method [18-20]. To achieve robustness, predictability, and scalability of the robotic swarm, we used a decentralized control multirobot cooperation strategy based on the principles of virtual physics. We use the term "virtual physics" because although these forces are defined only inside the control software, the robots act as though the forces are real. Physics-based systems, whether natural or virtual, can be analyzed using standard mathematical and physics theoretical principles. Such analysis facilitates a thorough understanding of the system and its predictability. When systems are predictable, it is straightforward to provide the necessary behavioral assurances that the swarm will perform as desired, even in the absence of human supervision [21]. The physics-based approach we adapted may lack some of the biomimetic intuition, but we gain much in terms of our analytic ability to evaluate and predict the collective's longterm behavior.

Zarzhitsky et al. [22] proposed physics based framework for distributed control of autonomous vehicles during localization of the source of a toxic plume. The problem statement in this paper explained that there are multiple chemical sources in the environment and the robots should locate all of them. Different from the case of one chemical source, locating several chemical sources using multirobot faces the following new problems: (1) how to partition the robots during a search to ensure that all sources are located in minimal time; (2) how to continue searching for other sources once a source has been found; (3) how to avoid refinding the same source. However, the existing methods of locating multiple chemical sources still has the following deficiencies: (1) a stationary plume environment is utilized to simulate the distribution of chemical plume in natural environment [23, 24], which is actually dynamic and turbulent; (2) a robot is regarded as a particle, that is to say, his physical shape is neglected $[13,24]$; (3) a sequential way is adopted to locate multiple chemical sources, that is to say, the method can locate only one chemical source at one run $[25,26]$.

This paper proposes a novel methodology-release strategy. One chemical source is located at a time and a forbidden area is set and published to the searching robots. Thus, they can release from the forbidden area and proceed with the search process, eventually finding other existing plumes and sources. In our previous work $[27,28]$, the virtual physics has been used for chemical localization. The proposed results are limited to simplified models of one or two chemical sources dispersion, with the same release rate to the source and without obstacles. In this paper, an approach based on the generalization of the virtual physics with a release strategy to the more difficult case of a turbulent chemical plume is proposed. Compared with the existing methods in the literature that work with localization of multiple chemical sources, our method has the following advantages: (1) a dynamic and turbulent plume environment is utilized to simulate the distribution of chemical plume in natural environment using computational fluid dynamics (CFD) software, Fluent (Fluent, Inc.). The plume from varied sources is converged together, which makes the robots unable to tell which source the plume they detected comes from; (2) the robot used in this paper has a physical shape and foot print of finite size; (3) the method can locate multiple chemical sources at one run using parallel search by more than one group. In addition, the physics-based approach we adapted can be analyzed using standard mathematical and physics theoretical principles. Such analysis facilitates a thorough understanding of the system and its predictability. So, this method facilitates transplanting the control strategy on the true swam robots in real-world environments.

\section{Benchmark Cases of Multiple Chemical Sources}

The benchmark cases of multiple chemical sources include the following attributes: the characterization and distribution of sources, the presence of dead space (involving no perceptible gradient), the characterization and distribution of obstacles, presence of time-varying background flow, and the model of robots. The characterization and distribution of the sources in the field provide sources are occluded by other sources with lesser, greater, or equal intensity. Dead space is occupied to determine the impact of an imperceptible gradient on search performance. The characterization and distribution of obstacles in the field block the chemical plume propagation and the robot motions to determine the impact of search performance in obstructed environment. The presence of time-varying background flow makes the distribution of the plume still more dynamic and turbulent. The robot used in this paper is not regarded as a particle, but has a physical shape and foot print of finite size, so the robots must avoid collisions with other robots in addition to avoiding obstacles in the obstructed environment.

\subsection{Dynamic Plume Models of Multiple Chemical Sources.} Three plume models used in the paper are obtained from Liu and $\mathrm{Lu}$ [29]. The chemical plume propagation was simulated using computational fluid dynamics (CFD) software, Fluent (Fluent, Inc.). The plume data produced by Fluent were then imported into Matlab (Mathworks, Inc.) and used by simulated mobile robots for plume-tracing behavior simulations. More efforts were engaged and the simulation framework was improved to simulate scenarios including multiple chemical 


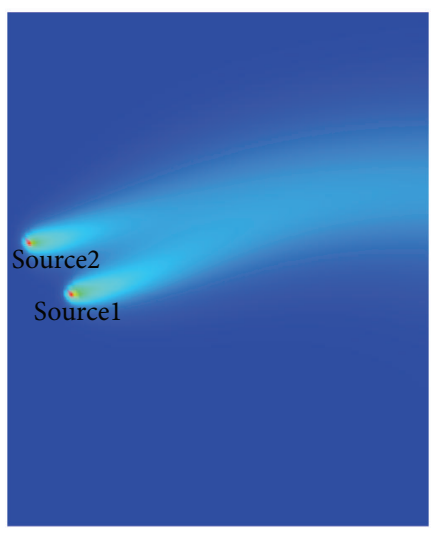

(a)

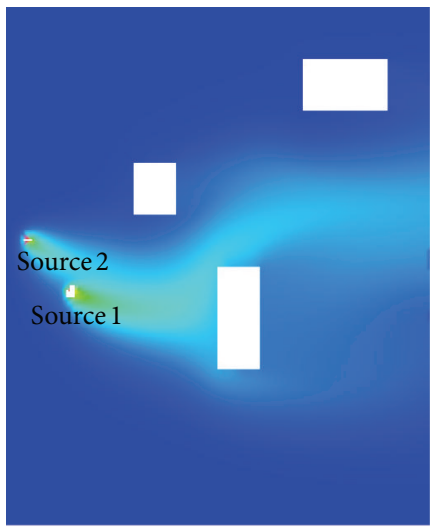

(b)

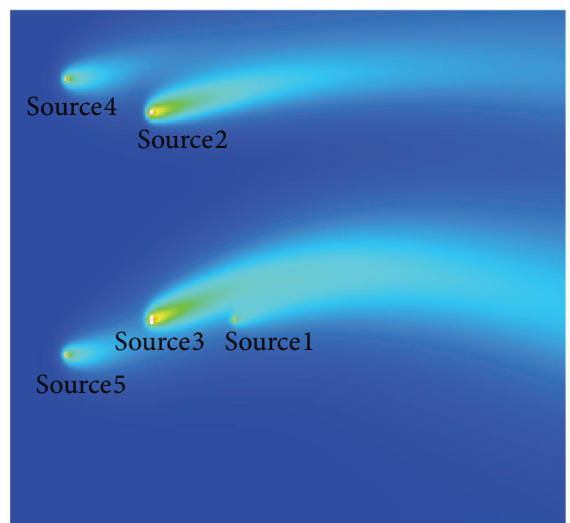

(c)

FIGURE 1: Instantaneous distribution of turbulent plume (a) Case 1, (b) Case 2, and (c) Case 3.

sources environments with different chemical release rate as well as multiple robots. The first scenario includes two chemical sources located at $(1,11)$ and $(3,9)$, respectively, in the environment with of size $20 \mathrm{~m} \times 20 \mathrm{~m}$, called Case 1 , and the second scenario called Case 2, adds three obstacles besides the same two chemical sources as Case 1 and the third scenario includes five chemical sources located at $(8,6),(5$, $12),(5,6),(2,13)$, and $(2,5)$, respectively, in the environment with size of $15 \mathrm{~m}$ in the $X$ direction and $20 \mathrm{~m}$ in the $Y$ direction, called Case 3. In Case 1, two chemical sources have the same release rates $500 \mathrm{~kg} / \mathrm{m}^{3}-\mathrm{s}$. In Case 2 , three obstacles with $(7 \pm 1,13 \pm 1),(11 \pm 1,8 \pm 2)$, and $(16 \pm 2,17 \pm 1)$ as their vertices block the chemical plume propagation and the robot motions. In Case 3, the release rates of five chemical sources are not the same: source 1 has the lowest release rate $150 \mathrm{~kg} / \mathrm{m}^{3}$-s; source 2 and source 3 have the same highest release rate $500 \mathrm{~kg} / \mathrm{m}^{3}-\mathrm{s}$; source 5 and source 6 have the same release rate $300 \mathrm{~kg} / \mathrm{m}^{3}$-s. Figure 1 shows instantaneous distribution of turbulent plumes of the three cases. The higher concentration is shown brighter in Figure 1. In color, highest concentration is shown as red and then yellow, green, and finally white as can be seen from Figure 1. The airflow entered into the left-hand side boundary of the domain at a constant $5 \mathrm{~m} / \mathrm{s}$ velocity and existed from the right-hand side boundary of the domain. The wind direction changes between $-22.5^{\circ}$ and $22.5^{\circ}$ measured along the $x$ axis of Figure 1 is governed by the equation of " $22.5 * \sin (10 * t)$ ", where $t$ is the time steps. The combined settings of the chemical sources and wind thus produce snapshots of complicated but realistic transient wind and plume propagations and distributions at different time steps with one-second increments for robot plume tracing and source localization to take place and are retained the same to be repeated for the required studies [30].

2.2. Model of the Robots. Consider a group of $N$ simulated mobile robots, moving in $R^{2}$ that are labeled as $R_{1}, R_{2}, \ldots$, $R_{N}$. Each robot $R_{i}(i=1, \ldots, N)$ is able to communicate with the other robots in his group. The simulated robots are able

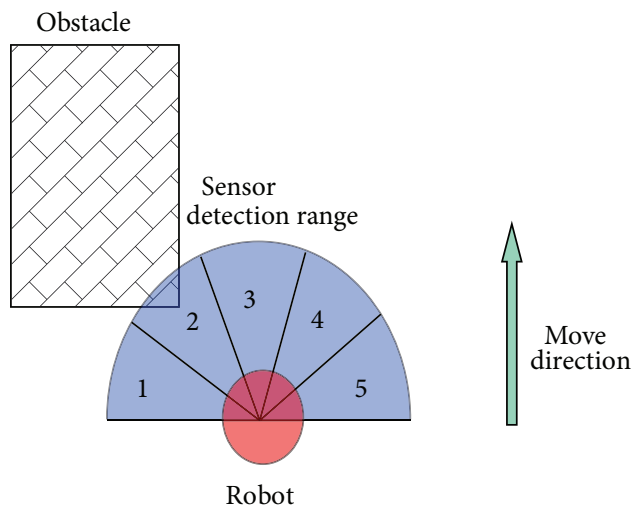

Figure 2: Configuration of the ultrasonic sensors of the robot.

to sense the chemical concentration and the wind velocity (speed and direction). The wind and chemical concentration information can always be "measured" by the robot wherever it is by reading in the data from the data file or interpolating among nodes if needed. Because the robot used in this paper has a physical shape and foot print of finite size, the robot must avoid obstacles in the obstructed environment. The robot has five ultrasonic sensors; as each ultrasonic sensor covers a $36^{\circ}$ angle; five sensors cover totally $180^{\circ}$ area in front of this robot which is enough for the robot to detect objects at the front. The configuration of ultrasonic sensors is illustrated in Figure 2.

As seen from Figure 1, the distribution of chemical plume we use is actually dynamic and turbulent. In Case 2 (see Figure 1(b)), the obstacles block the chemical plume propagation, make the plume more diversified and block the robot motions, make the search more difficult. In Case 3 (see Figure 1(c)), the variable intensities sources in the field are occluded by other sources with lesser, equal or greater intensity and provide the challenge in multisource localization because when sources of unequal intensity are 


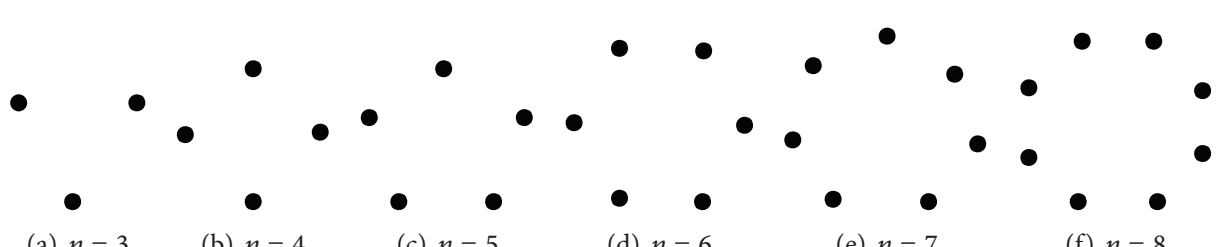
(a) $n=3$
(b) $n=4$
(c) $n=5$
(d) $n=6$
(e) $n=7$
(f) $n=8$

Figure 3: Six usual formations with different robot number $n$.

within range of a robot, the robot is likely to locate the stronger source and overlook the weaker source.

According to the benchmark cases of multiple chemical sources we use, there are the following problems that need to be solved in the rest of the paper: how to allocate the robots which have physical shape during a search to ensure that all sources are simultaneously located in minimal time; how to reset the robots to continue searching for other sources once a source has been located; and how to avoid the robots relocating the same source that has located and continue searching the other sources.

\section{Multirobot Cooperation Method Based on Virtual Physics with a Release Strategy}

In this section, a multirobot cooperation method based on virtual physics is presented to solve the problem of multiple chemical sources localization. Virtual physics (VP) provides a distributed control of mobile robots in sensor network. Every robot observes the environment, notes the position of nearby robots, and then computes virtual forces imposed upon it. After taking a vector sum of all forces on the robot, it takes derivatives to convert the net force into a velocity vector for the robot's next move. Generally, the control force includes two kinds of effort, which are virtual structure force $F(V S)$ and virtual goal force $F(V G)$. Then, taking a vector sum of all forces, that is, $F=F(\mathrm{VS})+F(\mathrm{VG})$, it converts the net force into a velocity vector for the robot's next move.

3.1. Virtual Structure Force. F(VS) is a function of distance between the neighboring robots and may depend on the pairwise combination of the vehicles, but $F(\mathrm{VS})$ does not depend on time.

3.1.1. Virtual Structure Force Based on Regular Polygon Formation. This paper adopted virtual structure force in [31], which makes the robotic swarms keep a regular polygon formation. The input force $\left(F_{x k}^{\prime}(\mathrm{VS}), F_{y k}^{\prime}(\mathrm{VS})\right)$ to the $K$ th $(k=$ $1,2,3, \ldots, n)$ robot for regular polygon formation based on the virtual structure can be stated as follows:

$$
\begin{aligned}
F_{x k}^{\prime}(\mathrm{VS})= & \sum_{i=1, i \neq k}^{n} k_{r} \frac{q_{k} q_{i}}{d_{k i}^{2}} \cos \left(\theta_{k i}\right) \\
& -k_{s}\left(x_{k}-x_{c}\right)\left(\left(x_{k}-x_{c}\right)^{2}+\left(y_{k}-y_{c}\right)^{2}-\alpha^{2}\right),
\end{aligned}
$$

$$
\begin{aligned}
F_{y k}^{\prime}(\mathrm{VS})= & \sum_{i=1, i \neq k}^{n} k_{r} \frac{q_{k} q_{i}}{d_{k i}^{2}} \sin \left(\theta_{k i}\right) \\
& -k_{s}\left(y_{k}-y_{c}\right)\left(\left(x_{k}-x_{c}\right)^{2}+\left(y_{k}-y_{c}\right)^{2}-\alpha^{2}\right),
\end{aligned}
$$

where $k_{r}$ and $k_{s}$ are positive gain, $q_{k}$ and $q_{i}$ are the $K$ th and $i$ th electric charges $\left(q_{k}=q_{i}=1\right.$ in this paper), $d_{k i}$ is the distance between them, $\left(x_{k}, y_{k}\right)$ is the position of robot $k$, and

$$
\cos \left(\theta_{k i}\right)=\frac{x_{k}-x_{i}}{\left|d_{k i}\right|}, \quad \sin \left(\theta_{k i}\right)=\frac{y_{k}-y_{i}}{\left|d_{k i}\right|} .
$$

The force defined in (1) makes the robot move toward the circle with center $\left(x_{c}, y_{c}\right)$ and radius $\alpha$ when $\left(x_{k}, y_{k}\right) \neq$ $\left(x_{c}, y_{c}\right)$ and form a regular polygon.

We obtained united vector as follows:

$$
\left(F_{x k}(\mathrm{VS}), F_{y k}(\mathrm{VS})\right)=\frac{\left(F_{x k}^{\prime}(\mathrm{VS}), F_{y k}^{\prime}(\mathrm{VS})\right)}{\left\|\left(F_{x k}^{\prime}(\mathrm{VS}), F_{y k}^{\prime}(\mathrm{VS})\right)\right\|}
$$

Six usual structures corresponding to different robot numbers on the basis of virtual structure force $\left(F_{x k}(\mathrm{VS})\right.$, $\left.F_{y k}(\mathrm{VS})\right)$ are illustrated in Figure 3.

For swarm formation of the robots, a virtual robot is considered at the center of the circle while the robots are placed on the circle around it. Therefore, $\left(x_{c}, y_{c}\right)$ in (1) should be replaced by $\left(x_{v}, y_{v}\right)$ that is the coordinate of the virtual robot. In this paper, the virtual robot was considered as a leader who received the sensor data (including chemical concentration, wind speed, and wind direction) available on robot $k(k=1,2,3, \ldots, n)$, processed the data, and made the motion decision.

3.1.2. Virtual Structure Force Based on Obstacle Avoidance. Because robots have a physical shape and foot print of finite size and cannot occupy the same position at the same time. Thus, robots must avoid collisions with other robots in addition to avoiding obstacles in the environment. In this paper, we adopted the simple reactive wall-following obstacle avoidance method which requires less computation and does not require a prior knowledge of the environment and past history of robot navigation.

Based on the sensory information from the ultrasonic sensors illustrated in Figure 2, if the robot detects an object within its alarm distance, it adjusts the movement direction to 
detour around that object until no obstacles are detected and then the robot will continue moving forward. The obstacle avoidance force is defined bellow.

Let $D$ be the distance readings from the five ultrasonic sensors:

$$
\begin{gathered}
D=\left\{d_{i}(t)\right\}, \quad i=1,2,3,4,5, \\
d_{i}(t)= \begin{cases}1, & d_{i}(t)<d_{0} \\
0, & \text { otherwise, }\end{cases}
\end{gathered}
$$

where $d_{0}$ is alarm distance.

The deflection angle of the move direction of the robot $\theta_{b}$ is defined as follows:

$$
\theta_{b}= \begin{cases}-90^{\circ} & d_{3}(t)=1 \\ -30^{\circ} & d_{2}(t)=1, d_{3}(t)=0 \\ -15^{\circ} & d_{1}(t)=1, d_{2}(t)=0, d_{3}(t)=0 \\ 30^{\circ} & d_{4}(t)=1, d_{3}(t)=0 \\ 15^{\circ} & d_{5}(t)=1, d_{4}(t)=0, d_{3}(t)=0 \\ 0 & \text { otherwise. }\end{cases}
$$

Let $\widetilde{\theta}$ be the included angle between $F_{k}(\mathrm{VS})(k=1,2,3$, $\ldots, n)$ to the $K$ th $(k=1,2,3, \ldots, n)$ robot and positive axis of $x$; then the virtual structure force $\left(F_{x k}(\mathrm{VS}), F_{y k}(\mathrm{VS})\right)$ to the $K$ th $(k=1,2,3, \ldots, n)$ robot will be modified by

$$
\begin{aligned}
& F_{x k}^{\prime \prime}(\mathrm{VS})=F_{k}(\mathrm{VS}) \cos \left(\tilde{\theta}+\theta_{b}\right), \\
& F_{y k}^{\prime \prime}(\mathrm{VS})=F_{k}(\mathrm{VS}) \sin \left(\widetilde{\theta}+\theta_{b}\right) .
\end{aligned}
$$

3.2. Virtual Goal Force. F(VG), a task-specific force that is responsible for directing the swarm toward a goal location, is typically computed using the sensor data available on robot $i$ at time $t$ and therefore depends on both time and the individual robot that is computing the goal force. We put the virtual goal force only on virtual robot.

\subsubsection{Virtual Goal Force Based on Different Plume-Tracing Algorithms}

Chemotaxis. The gradient strategy simply follows the chemical gradient, so the direction of the largest chemical concentration is the goal direction. The virtual robot receives the sensor data available on robot $k(k=1,2,3, \ldots, n)$, chooses the robot $j$ who has the highest concentration and moves toward it a distance of step length $s_{1}$. The virtual goal force is defined as follows:

$$
F(\mathrm{VG})=\frac{X_{j}(t)-X_{v}(t)}{\left\|X_{j}(t)-X_{v}(t)\right\|},
$$

where $X_{v}(t)$ and $X_{j}(t)$ are positions of virtual robot and robot $j$ at time step $t$, respectively. $\|\cdot\|$ represents the Euclidean norm operator.

Anemotaxis. The intuition behind the anemotaxis is to move the lattice upstream while keeping the robots inside the plume. If the gas concentration of a robot sensed is lower than a given threshold $\rho_{T}$, the robots perform chemotaxis. Otherwise, if the gas concentrations all robots sensed exceeded $\rho_{T}$ and the wind velocities were not all zero, the virtual robot receives the sensor data available on robot $k(k=$ $1,2,3, \ldots, n)$, records all the wind velocities: $\vec{v}_{1}, \vec{v}_{2}, \ldots, \vec{v}_{n}$, and calculates the average wind velocity $\overline{\vec{v}}=(1 / n) \sum_{i=1}^{n} \vec{v}_{i}$. Then, the virtual robot chooses upwind direction $-\vec{v}$ and moves toward it a distance of step length $s_{1}$. The virtual goal force is defined as follows:

$$
F(\mathrm{VG})=-\frac{\overline{\vec{v}}}{\|\overline{\vec{v}}\|} .
$$

3.2.2. Virtual Goal Force Based on Repulsion Force in Parallel Search. To make search faster, we can use parallel search by several groups' robots. Parallel search logically makes searching time shorter. Several groups of robots run and find chemical sources separately. However, the robots from one group are movable obstacles because robots have a physical shape; the other group must avoid collisions with them. In order to guarantee the positions of one group's robots to be away from the other, we assume that there is a repulsion force $f_{v}$ between the virtual robots of different groups. The repulsion force $f_{v}$ acting on the virtual robot of group $k$ by other groupsis defined as follows:

$$
f_{v}= \begin{cases}\sum_{i=1, i \neq k}^{m} \frac{k_{v}\left(X_{i v}-X_{k v}\right)}{\left\|X_{i v}-X_{k v}\right\|^{2}} & \\ \cdot\left(\frac{1}{\left\|X_{i v}-X_{k v}\right\|}-\frac{1}{5 \alpha}\right), & \left\|X_{i v}-X_{k v}\right\|<5 \alpha \\ 0, & \text { otherwise, }\end{cases}
$$

where $X_{k v}$ and $X_{i v}$ are the positions of virtual robots of group $k$ and group $i$, respectively. $m$ is the number of groups in parallel search. $\|\cdot\|$ represents the Euclidean norm operator. Then, the virtual goal force of group $k$ is modified by

$$
F_{k}^{\prime}(\mathrm{VG})=F_{k}(\mathrm{VG})+f_{v} \text {. }
$$

3.2.3. Virtual Goal Force Based on Release Strategy. Once the robots of group $i(i=1,2,3, \ldots, m)$ first locate a source and declare it, we set a circular forbidden area with its center at the virtual robot of group $i$ and radius $r_{v T}$. Forbidden area setting has two objectives: one is to ensure that the robots within the forbidden area are released and thus have the opportunity to find the other chemical source; the other is to ensure that the robots outside the forbidden area do not relocate this chemical source.

The circular forbidden area with its center at the virtual robot of group $i$ and radius $r_{v T}$ is set as follows:

$$
\left(x-x_{i v}\right)^{2}+\left(y-y_{i v}\right)^{2} \leq r_{v T}^{2}
$$

where $\left(x_{i v}, y_{i v}\right)$ is the position of the virtual robot of group $i$ and $r_{v T}\left(\alpha<r_{v T}<5 \alpha\right)$ is a parameter. 


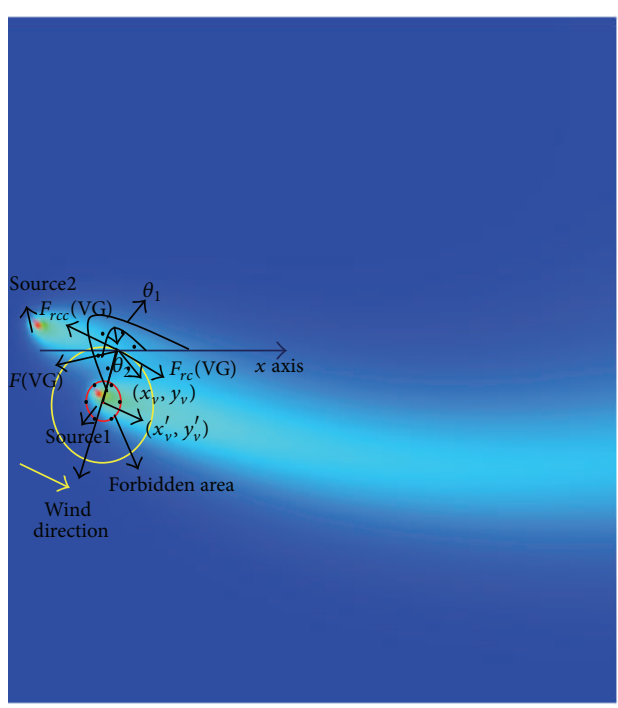

FIGURE 4: Three kinds of goal forces acting on the virtual robot of the group $i$.

In order to release from the forbidden area, the virtual robot of the group $i$ can move vertical to the wind direction (left or right). The possibility of robot movement at the left or right side is equal. This behavior is similar to the silkworm moth "cast" across the wind. In this moment, the goal force acting on the virtual robot of the group $i$ can be modified as follows:

$$
\begin{aligned}
& f_{\text {release_left }}^{\prime \prime}(\mathrm{VG})=\left(\overline{\vec{v}}_{x},-\overline{\vec{v}}_{y}\right), \\
& f_{\text {release_right }}^{\prime \prime}(\mathrm{VG})=\left(-\overline{\vec{v}}_{x}, \overline{\vec{v}}_{y}\right) .
\end{aligned}
$$

Let us normalize it as follows:

$$
F_{\text {release }}^{\prime \prime}(\mathrm{VG})=\frac{f_{\text {release }}^{\prime \prime}(\mathrm{VG})}{\left\|f_{\text {release }}^{\prime \prime}(\mathrm{VG})\right\|} .
$$

For making the robots of group $i$ quickly out of the forbidden area (indicated by a red circle in Figure 4), we specify the step length $s_{1}$ of the virtual robot and the robots of the group $i$ are $3 \times r_{v T}$ such that the robots of group $i$ can move out of the forbidden area by one step. Then, the virtual robot restores to the original step length. At the moment, there are three kinds of goal forces we assumed acted on the virtual robot of the group $i$ : $F(\mathrm{VG}), F_{r c}(\mathrm{VG})$, and $F_{r c c}(\mathrm{VG})$ as can be seen from Figure 4 , where $F(\mathrm{VG})$ is defined by plume-tracing algorithm (formulas (7) and (8)). $F_{r c}(\mathrm{VG})$ and $F_{r c c}(\mathrm{VG})$ are normalized clockwise force and counter clockwise force, respectively, which are defined as follows:

$$
F_{v r}^{\prime}(\mathrm{VG})=\frac{f_{x v r}}{\left|f_{v r}\right|} \vec{i}+\frac{f_{y v r}}{\left|f_{v r}\right|} \vec{j} .
$$

The following equations express the direction of the rotary force in two different conditions:

$$
\begin{aligned}
& f_{x v r}=f_{x v r c}, \quad f_{y v r}=f_{y v r c} \quad \theta_{1} \leq \theta_{2}, \\
& f_{x v r}=f_{x v r c c}, \quad f_{y v r}=f_{y v r c c} \quad \theta_{1}>\theta_{2} \text {, }
\end{aligned}
$$

where $\left(f_{x v r c}, f_{y v r c}\right)$ is clockwise force and $\left(f_{x v r c c}, f_{y v r c c}\right)$ is counter clockwise force. $\theta_{1}$ is the angle between the positive direction of $x$-axis and the directed line connected the positions of the virtual robot of group $i$ in and out of the forbidden area (the start for the current position $\left(x_{v}, y_{v}\right)$, the end for the previous position $\left.\left(x_{v}^{\prime}, y_{v}^{\prime}\right)\right)$ in counterclockwise direction. And $\theta_{2}$ is the angle between the positive direction of $x$-axis and the goal force $F(\mathrm{VG})$ of the group $i$ in counterclockwise direction:

$$
\begin{gathered}
f_{\text {xvrc }}=-\left(y_{v}^{\prime}-y_{v}\right), \\
f_{y v r c}=\left(x_{v}^{\prime}-x_{v}\right), \\
f_{x v r c c}=\left(y_{v}^{\prime}-y_{v}\right), \\
f_{y v r c c}=-\left(x_{v}^{\prime}-x_{v}\right),
\end{gathered}
$$

where $\left(x_{v}, y_{v}\right)$ and $\left(x_{v}^{\prime}, y_{v}^{\prime}\right)$ are the positions of the virtual robot of group $i$ in and out of the forbidden area by one step, respectively.

As shown in Figure 4, when $\left|\theta_{1}-\theta_{2}\right| \leq \pi / 2$, the group $i$ by force $F_{v r}(\mathrm{VG})$ can successfully bypass source 1 and locate source 2. It should be noted that once the virtual robot of group $i$ chose a rotary method (clockwise or counter clockwise), it should keep on until it escaped from source 1. When $\left|\theta_{1}-\theta_{2}\right|>\pi / 2$, the group $i$ by force $F(\mathrm{VG})$ can successfully move away from source 1 . The method of movement direction chosen by virtual robot of the group $i$ is shown in Figure 5.

In order to avoid the robots relocating the same source that has been located and continue searching the other sources, when a group of robots move around a forbidden area, that is, the distance between the virtual robot of this group and the center of the forbidden area is less than $3 \times$ $r_{v T}$, the virtual robot will also choose a movement direction according to Figure 5. The robots will not stop until all chemical sources are found or after maximum iterations (in our simulation, maximum iterations is 2000).

3.3. Movement of the Robots of a Group. The discrete-time model of the virtual robot movements can be stated as

$$
X_{v}(t+1)=X_{v}(t)+s_{1} \cdot F(\mathrm{VG}) \text {, }
$$

where $X_{v}(t+1)$ and $X_{v}(t)$ are positions of the virtual robot at time step $t+1$ and $t$, respectively.

As the virtual robot moved to a new position, the robots would also move a distance of step length $s_{2}$ under the action of the virtual structure force $F(V S)$.

So, the discrete-time model of the robot movements can be stated as

$$
X_{k}(t+1)=X_{k}(t)+s_{2} \cdot F_{k}(\mathrm{VS}) \text {, }
$$

where $X_{k}(t+1)$ and $X_{k}(t)$ are position of the robot $k(k=1$, $2,3, \ldots, n)$ at time step $t+1$ and $t$, respectively. 
TABLE 1: Parameters of algorithm with release strategy.

\begin{tabular}{lcccccccc}
\hline$k_{s}$ & $\alpha$ & $k_{r}$ & $s_{1}$ & $s_{2}$ & $d_{0}$ & $n_{s}$ & $\Phi_{T}$ & $r_{v T}$ \\
\hline 0.0001 & $0.3[\mathrm{~m}]$ & 5 & $0.06[\mathrm{~m}]$ & $0.12[\mathrm{~m}]$ & $0.8[\mathrm{~m}]$ & 10 & $0.1\left[\mathrm{~kg} / \mathrm{m}^{2} \cdot \mathrm{s}\right]$ & $0.6[\mathrm{~m}]$ \\
\hline
\end{tabular}

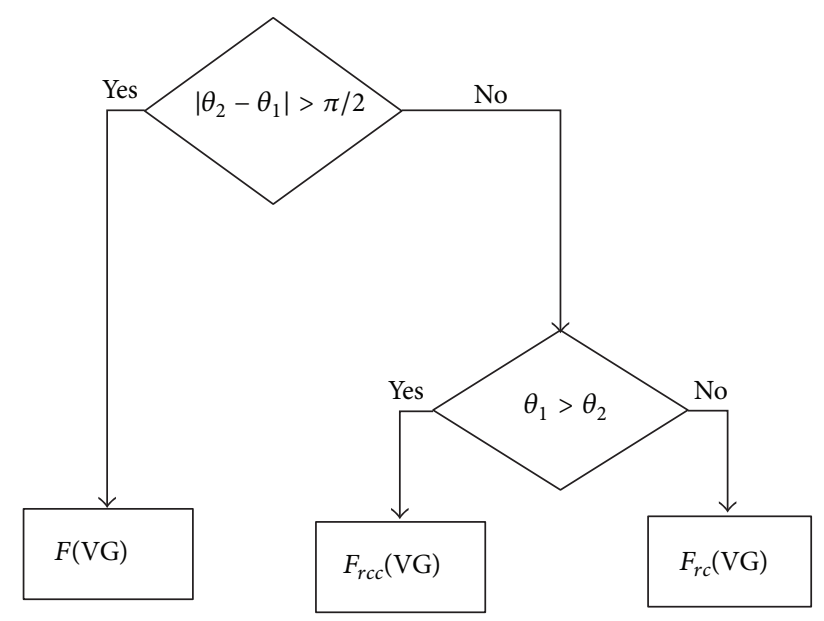

FIGURE 5: The method of movement direction chosen by virtual robot of the group $i$.

\section{Experiments and Analysis: Scenarios with Two Chemical Sources}

Experiments are performed on two scenarios with two chemical sources (Case 1 and Case 2). Both types of experiments provided successful solutions. One aim of these experiments is to investigate the impacts caused by the random localization of the robots when they are released in an environment with no prior knowledge available to commence plume tracing. The other is to test the search efficiency of the proposed multirobot system based virtual physics force at different frequencies of wind direction/speed and methane release.

Here, three positions ( $x$ coordinate, $y$ coordinate of the virtual robot) are randomly chosen, $(18,10)$ called $\mathrm{P} 1,(18,4)$ called P2, and $(18,16)$ called P3. Three kinds of frequencies of wind direction/speed and release frequency of two methane sources are used: standard frequency, twice frequency, and treble frequency. According to the results in [27], we choose a group of 6 robots composed of hexagonal grids to multiple sources localization.

At the beginning, when the robots of a group find no plume, the robots would perform passive monitoring [10] to find plume, that is, the robots remain stationary and wait for a chemical plume to intersect the robots' current location. The male silkworm moth employs this strategy. When trying to detect the plume of pheromone released by the female silkworm moth, the male moth waits, head into the wind in an exposed position, until it detects the pheromone. If one robot of the group find plume, the robots will use chemotaxis to trace the gas plume. When arriving at the source region, because of the balance of the inner structure force and external goal force, the robots get bogged down and become
TABLE 2: Comparisons on time consumption used by robots at three different frequencies with three different initial positions in Case 1.

\begin{tabular}{lcccc}
\hline & $\begin{array}{c}\text { Standard } \\
\text { frequency }\end{array}$ & $\begin{array}{c}\text { Twice } \\
\text { frequency }\end{array}$ & $\begin{array}{c}\text { Treble } \\
\text { frequency }\end{array}$ & Average time \\
\hline P1 & 523 & 529 & 873 & 642 \\
P2 & 500 & 835 & 941 & 759 \\
P3 & 1053 & 862 & 1146 & 1020 \\
\hline Average time & 692 & 742 & 987 & \\
\hline
\end{tabular}

a circle surrounding the potential source. If the total mass flux measured by the sensor grid consistently exceeds some small, empirically-determined threshold $\Phi_{T}$ in a given number of steps $n_{s}$, we decide that the chemical source is located.

4.1. Parameters of the Algorithm. By simulating extensive numerical search trials for each parameter, we choose the parameter given by Table 1 .

4.2. Performance Metrics. Two measures are adopted to evaluate the performance of an algorithm in this paper. The first is the success rate which reflects the ratio of the times that successfully locate all chemical sources to the total runtimes. The second is the time consumption of localizing all chemical sources by the robots with proposed strategy.

\subsection{Simulation Results}

4.3.1. Simulation Results in Case 1. Firstly, we give the simulation results in Case 1 . To get a better understanding of the effect that the control algorithm with release strategy has on the plume tracing task, we give a series of snap shots of the tracing chemical plumes process of the six robots of a group in Case 1 (see Figure 6). At the beginning, six robots (indicated by "O") and a virtual robot (indicated by "+") are distributed randomly in position P1. At the time $t=295 \mathrm{~s}$ (see Figure 6(a)), the robots trace the plume to the Source 1 with the control algorithm while preserving a stable hexagonal structure. Then, a circular forbidden area (indicated by a red circle) is set. At the time $t=298 \mathrm{~s}$ (see Figure 6(b)), the robots are released from forbidden area with release strategy and choose counterclockwise movement bypass the located Source 1. At last, the robots find the other source Source 2 and the search is complete (see Figure 6(c)).

Figures 7, 8 and 9 give the plume tracing paths of a group of robots using chemotaxis at three kinds of frequency with three initial positions, respectively, in Case 1. Table 2 gives the time consumption used by two sources locating at three different frequencies with three different initial positions.

Figures 7-9 and Table 2 tell us that the proposed search strategy using chemotaxis is effective and obtains $100 \%$ 


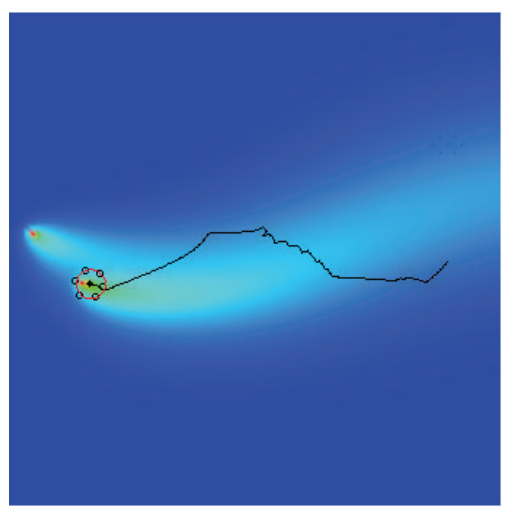

(a) $t=295 \mathrm{~s}$

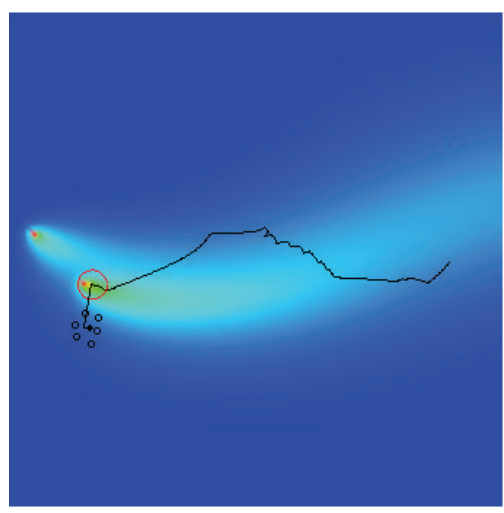

(b) $t=298 \mathrm{~s}$



(c) $t=455 \mathrm{~s}$

FIGURE 6: Search process by one group of six robots with release strategy.

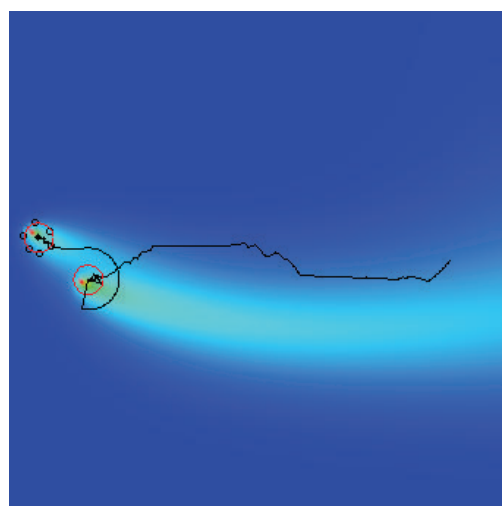

(a) Standard frequency

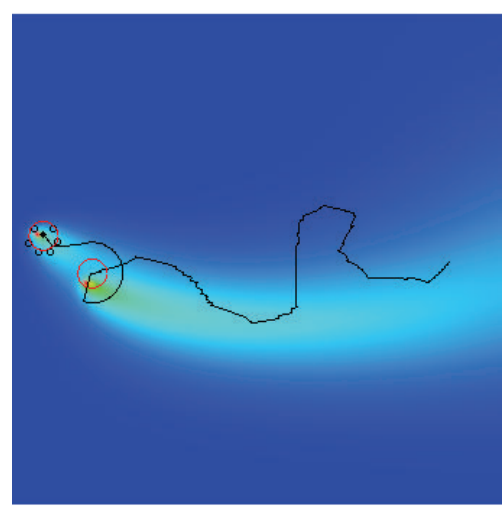

(b) Twice frequency

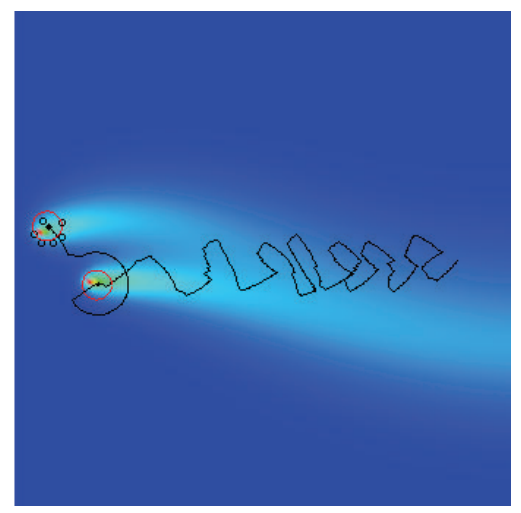

(c) Treble frequency

FIGURE 7: Search process by one group of six robots with release strategy at different frequencies with initial position P1.

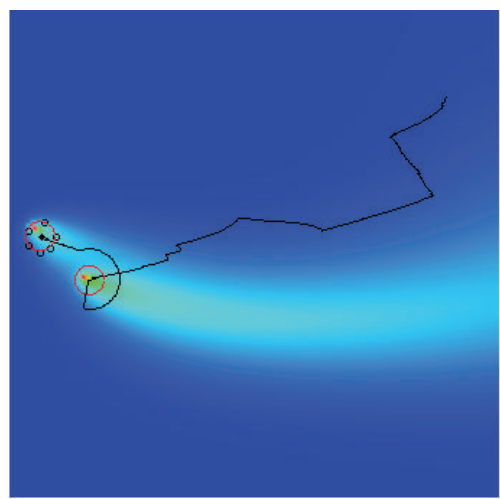

(a) Standard frequency

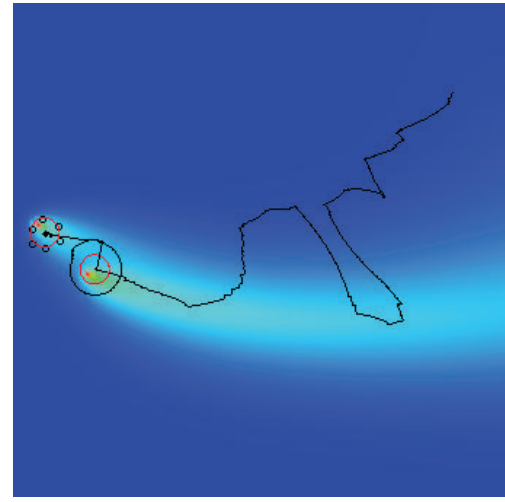

(b) Twice frequency

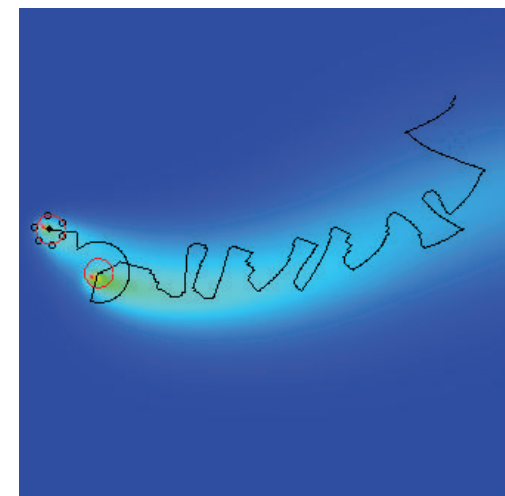

(c) Treble frequency

FIGURE 8: Search process by one group of six robots with release strategy at different frequencies with initial position P2.

success rate; however, with the increasing of the wind direction/speed frequency and methane release frequency, the time consumption used by the robots increases. The main reason is that the increasing of the wind direction/speed frequency and methane release frequency make the plume drift up and down drastically, the robots using the chemotaxis move towards the direction of the largest chemical concentration which make the tracing paths similar to the variation pattern of the wind direction which changes between $-22.5^{\circ}$ and $22.5^{\circ}$ governed by the equation of " $22.5 * \sin (10 * t)$." For example, the average time is $692 \mathrm{~s}$ at standard frequency, while the average search time is $987 \mathrm{~s}$ at treble frequency. 


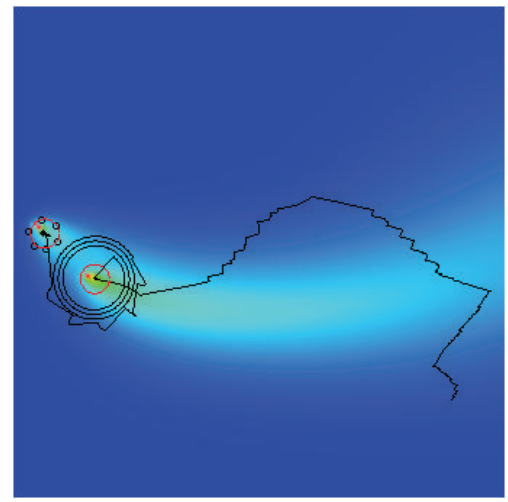

(a) Standard frequency

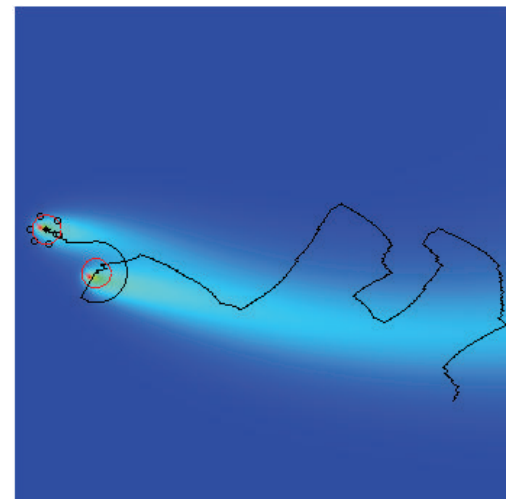

(b) Twice frequency

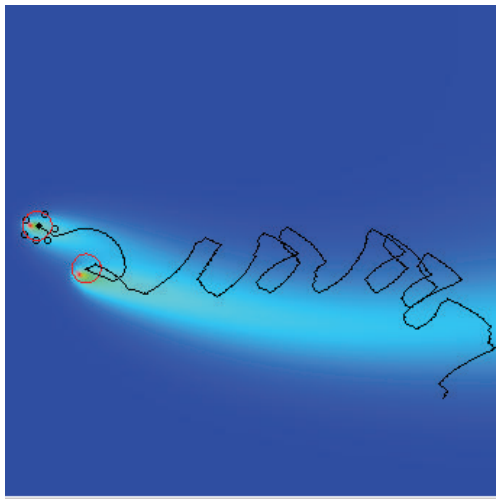

(c) Treble frequency

FIGURE 9: Search process by one group of six robots with release strategy at different frequencies with initial position P3.

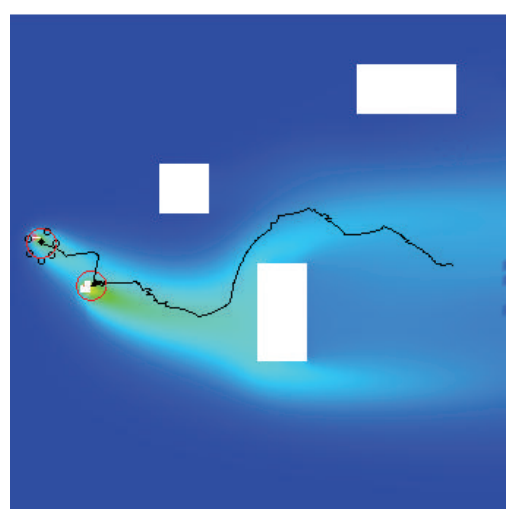

(a) Standard frequency

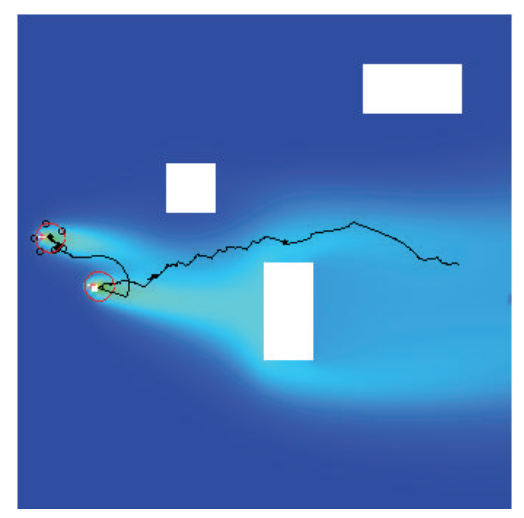

(b) Twice frequency

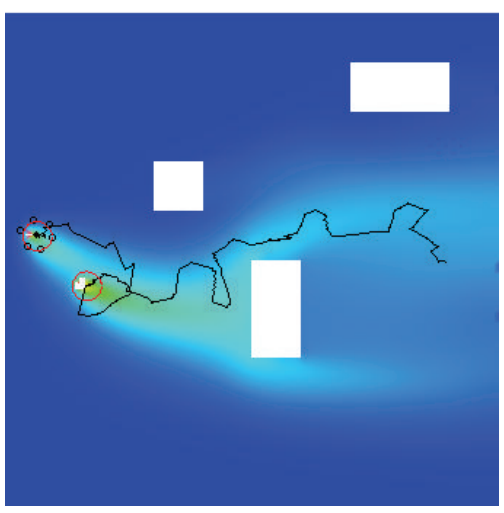

(c) Treble frequency

FIGURE 10: Search process by one group of six robots with release strategy at different frequencies with initial position P1.

Considering three different initial positions we had tested, Groups start out from P3 spent the most time to locate two sources and groups start from P1 spent the least time at three different frequencies of wind direction/speed and methane release. For example, the average time is $642 \mathrm{~s}$ with $\mathrm{P} 1,759 \mathrm{~s}$ with $\mathrm{P} 2$, and $1020 \mathrm{~s}$ with $\mathrm{P} 3$. The main reason is that P3 is farthest from two sources; the farther the initial positions from two sources the longer time should be spent to located them. So, different initial positions of two groups exert influence on the search efficiency.

4.3.2. Simulation Results in Case 2. The simulated mobile robots using the proposed control strategies were also tested in an obstacle-filled arena (Case 2). The obstacles block the chemical plume propagation, make the plume more diversified and block the robot motions, make the search more difficult.

Figures 10, 11 and 12 give the plume tracing paths of a group of robots using chemotaxis at three kinds of frequency with three initial positions respectively in Case 2. Table 3 gives the time consumption used by two sources locating at three different frequencies with three different initial positions.
TABLE 3: Comparisons on time consumption used by robots at three different frequencies with three different initial positions in Case 2.

\begin{tabular}{lcccc}
\hline & $\begin{array}{c}\text { Standard } \\
\text { frequency }\end{array}$ & $\begin{array}{c}\text { Twice } \\
\text { frequency }\end{array}$ & $\begin{array}{c}\text { Treble } \\
\text { frequency }\end{array}$ & Average time \\
\hline P1 & 600 & 518 & 621 & 580 \\
P2 & 986 & 585 & 591 & 721 \\
P3 & 809 & 882 & 530 & 740 \\
\hline Average time & 798 & 662 & 581 & \\
\hline
\end{tabular}

Figures 10-12 and Table 3 tell us that the proposed strategy is very effective and obtain $100 \%$ success rate even in Case 2. With the increasing of the wind direction/speed frequency and methane release frequency, the search time used by the robots slightly decrease (see Table 3 ). For example, the average time is $798 \mathrm{~s}$ at standard frequency, while the average search time is $581 \mathrm{~s}$ at treble frequency. The main reason is that the robots moved in an obstructed environment, obstacle avoidance is prior to the robots; in addition, with the increasing of the frequency, the obstacles make the distribution of the plume more diversified and more available 


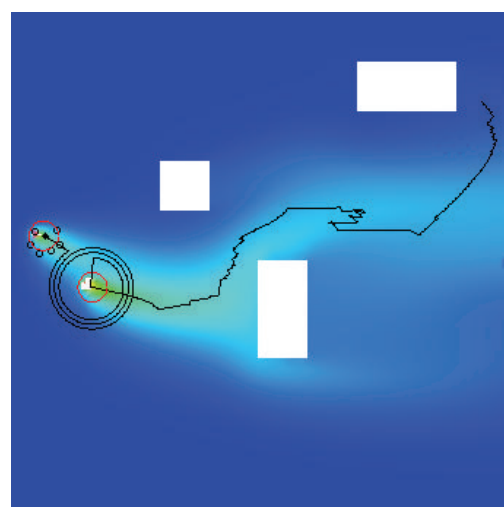

(a) Standard frequency

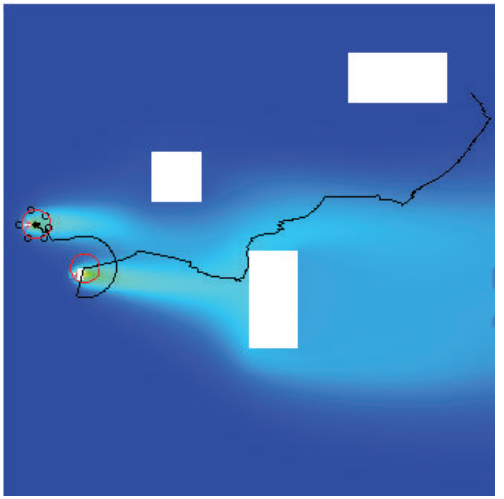

(b) Twice frequency

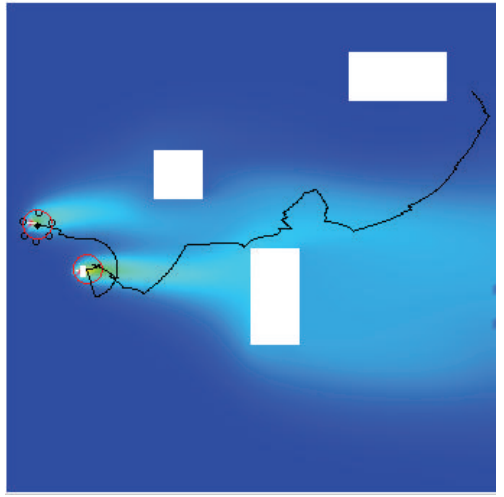

(c) Treble frequency

FIGURE 11: Search process by one group of six robots with release strategy at different frequencies with initial position P2.

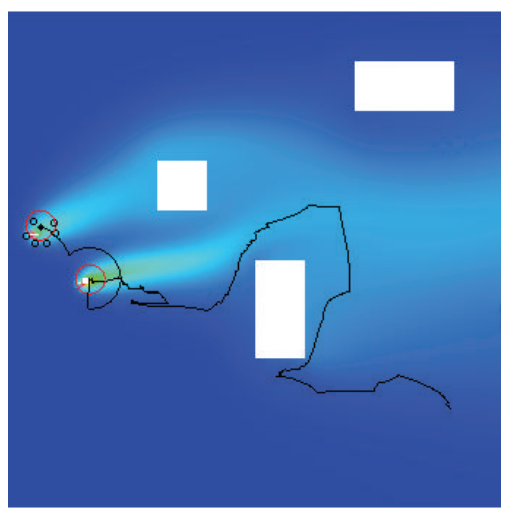

(a) Standard frequency

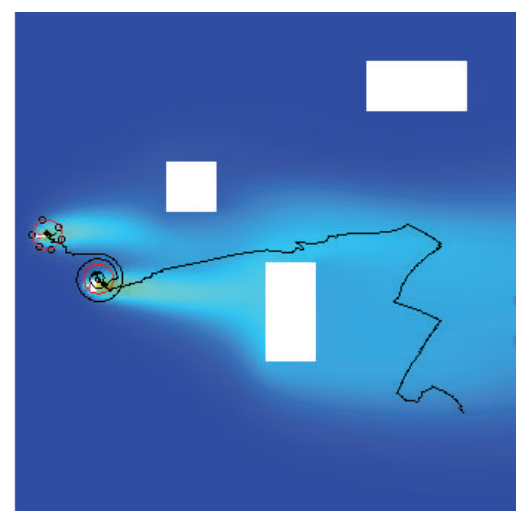

(b) Twice frequency

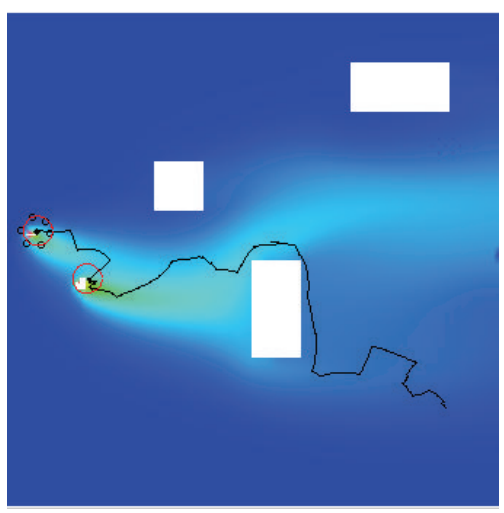

(c) Treble frequency

FIGURE 12: Search process by one group of six robots with release strategy at different frequencies with initial position P3.

to the robots which made the search time slightly decrease. Considering three kinds of initial position we had tested, the average time is $580 \mathrm{~s}$ with $\mathrm{P} 1,721 \mathrm{~s}$ with $\mathrm{P} 2$, and $740 \mathrm{~s}$ with P3. In advanced investigation, we deduced that the robots start from P2 and P3 are far from the sources and also behind obstacles; the group with this initial position should bypass the obstacle to locate the sources.

\section{Experiments and Analysis: Scenario with Five Chemical Sources}

5.1. Influence of Forbidden Area Radius $r_{v T}$ on the Proposed Strategy. The proposed release strategy uses the circular forbidden area to make the robots release from the located chemical sources; therefore, it is understandable that the radius $r_{v T}$ plays a crucial role in the performance of our strategy. This experiment performs an extensive analysis about the impact of $r_{v T}$ on the performance of our strategy at different frequencies of wind direction/speed and methane release, where the $r_{v T}$ is allowed to vary from $0.3 \mathrm{~m}$ to $0.6 \mathrm{~m}$.

To make search faster, we use parallel search by two groups' robots; then, there is a total of twelve robots used for five chemical sources localization in Case 3. Each group runs by itself. There is a repulsion force $f_{v}$ between the virtual robots of two groups which is defined by (9). According to the results in [28], more dispersed initial positions of two groups, the more effective by parallel search; we choose two positions, ( $x$ coordinate, $y$ coordinate of the virtual robot), $(18,5)$ and $(18,8)$ as initial positions of two groups. We use the same strategy of plume finding and chemical source declaration as the fourth section and use anemotaxis to trace the chemical plume.

We chose the parameter given by Table 4 .

To get a better understanding of the effect that the control algorithm with release strategy has on the plume tracing task in Case 3, we give a series of snap shots of the tracing chemical plumes process of two groups of robots with $r_{v T}=0.3 \mathrm{~m}$ (see Figure 13).

At the beginning, two groups of robots (indicated by yellow " $\bigcirc$ " and black " $\bigcirc$," resp.) are distributed randomly in $(18,5)$ and $(18,8)$. At the time $t=174 \mathrm{~s}$ (see Figure 13(a)), a group of robots indicated by black " $O$ " locate the first source Source 1. Then, a circular forbidden area (indicated by a red circle) is set. At the time $t=229 \mathrm{~s}$ and $t=270$ (see Figures 13(b) and 13(d), resp.), this group released from forbidden 
TABLE 4: Parameters of algorithm.

\begin{tabular}{lccccccc}
\hline$k_{s}$ & $\alpha$ & $k_{r}$ & $s_{1}$ & $s_{2}$ & $\rho_{T}$ & $\Phi_{T}$ & $n_{s}$ \\
\hline 0.0001 & $0.3[\mathrm{~m}]$ & 5 & $0.06[\mathrm{~m}]$ & $0.12[\mathrm{~m}]$ & $0.0095\left[\mathrm{~kg} / \mathrm{m}^{3}\right]$ & $0.1\left[\mathrm{~kg} / \mathrm{m}^{3}\right]$ & 10 \\
\hline
\end{tabular}

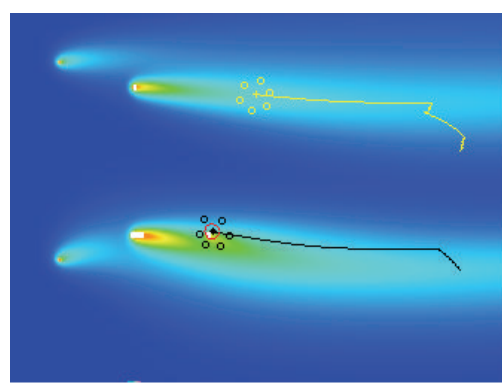

(a) $t=174 \mathrm{~s}$

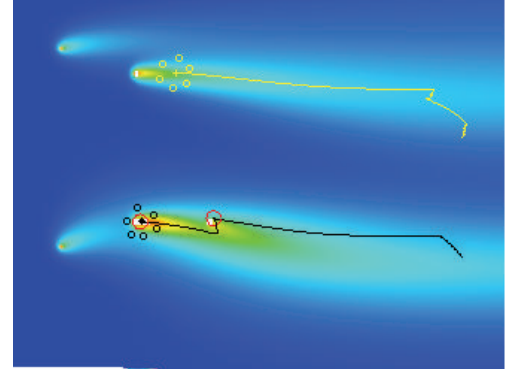

(b) $t=229 \mathrm{~s}$

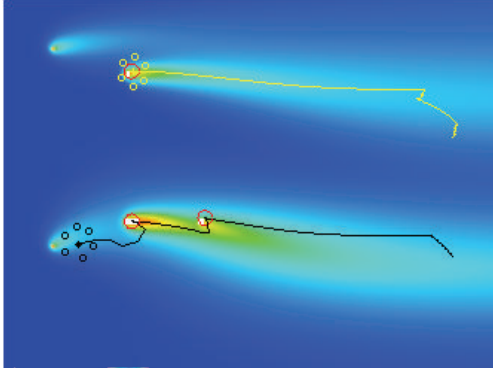

(c) $t=254 \mathrm{~s}$

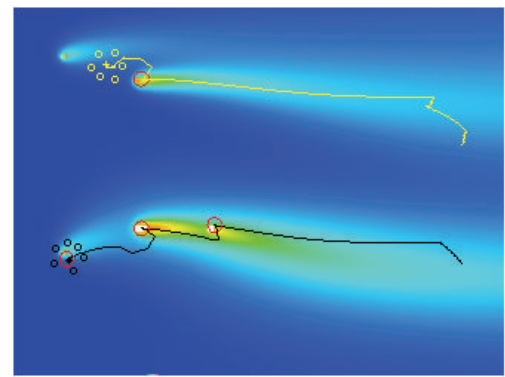

(d) $t=270 \mathrm{~s}$

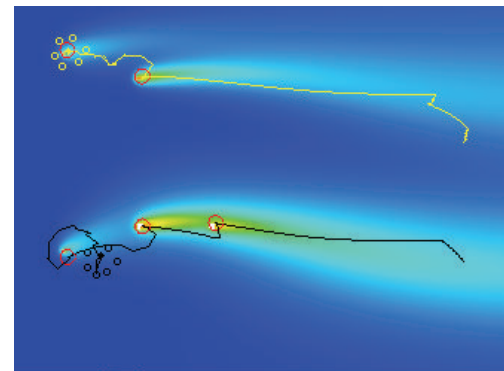

(e) $t=306 \mathrm{~s}$

FIGURE 13: Process of chemical source localization using anemotaxis at standard frequency.

area with release strategy and locate the Source 3 and the Source 5, respectively. At the time $t=254$ s (see Figure 13(c)), the other group of robots indicated by yellow "O" locate the Source 2. At last, the robots of this group locate the last source Source 4 and the search is ending (see Figure 13(e)).

Figures 14-16 give the plume tracing paths of two groups of robots using anemotaixs at three kinds of frequency with different radius $r_{v T}$, respectively in Case 3. Table 5 gives the search time used by sources locating.

It can be seen from Table 5 and Figures 14-16, when $r_{v T}=$ $0.3 \mathrm{~m}$, the proposed algorithm only locates four chemical sources at twice frequency (see Figure 15). The main reason is that, at this frequency, the group of robots indicated by yellow "O" using anemotaxis miss locating the source 2 when it is tracing plume and locates the source 4 (see Figure 15(a)). Forbidden area with smaller radius $r_{v T}$ and rotary force could not guarantee the group to escape from the plume of the source 4 thus finding the source downwind to the located source (anemotaxis makes the robots to move towards the upwind direction, not downwind direction). When $r_{v T}=$ $0.4 \mathrm{~m}$ or lager, the robots successfully locate the source 2 using rotary force and goal force (see Figures 15(b) and 15(c)). However, with the increasing of the radius $r_{v T}$, the stepsize of the robots moving out of the forbidden area increases that would make the robots that performing rotating search move out of the search area (see Figure 14(d)).

Therefore, the smaller forbidden area radius can enhance the exploitation of the robots and the larger radius can
TABLE 5: Comparisons on time consumption of sources located at three different frequencies with four different radii of taboo domain.

\begin{tabular}{lccccc}
\hline Number of sources located & 1 & 2 & 3 & 4 & 5 \\
\hline Standard frequency & & & & & \\
$\quad r_{v T}=0.3 \mathrm{~m}$ & 174 & 229 & 254 & 270 & 306 \\
$r_{v T}=0.4 \mathrm{~m}$ & 174 & 230 & 254 & 280 & 315 \\
$r_{v T}=0.5 \mathrm{~m}$ & 174 & 231 & 254 & 280 & 301 \\
$r_{v T}=0.6 \mathrm{~m}$ & 174 & 231 & 254 & 276 & 248 \\
Twice frequency & & & & & \\
$r_{v T}=0.3 \mathrm{~m}$ & 173 & 238 & 332 & 343 & - \\
$r_{v T}=0.4 \mathrm{~m}$ & 173 & 242 & 307 & 343 & 658 \\
$r_{v T}=0.5 \mathrm{~m}$ & 173 & 270 & 321 & 343 & 391 \\
$r_{v T}=0.6 \mathrm{~m}$ & 173 & 287 & 343 & 365 & 749 \\
Treble frequency & & & & & \\
$r_{v T}=0.3 \mathrm{~m}$ & 181 & 256 & 278 & 335 & 379 \\
$r_{v T}=0.4 \mathrm{~m}$ & 181 & 257 & 278 & 284 & 323 \\
$r_{v T}=0.5 \mathrm{~m}$ & 181 & 261 & 278 & 326 & 378 \\
$r_{v T}=0.6 \mathrm{~m}$ & 181 & 269 & 278 & 370 & 613 \\
\hline
\end{tabular}

enhance the exploration. With respect to time consumption, the proporite radius is 0.4 or 0.5 for parallel search by two groups; for one group search, the proporite radius is 0.6 or larger (see Figure 15(d)) which can make the paths of two groups interlaced together. In consideration of the possibility 


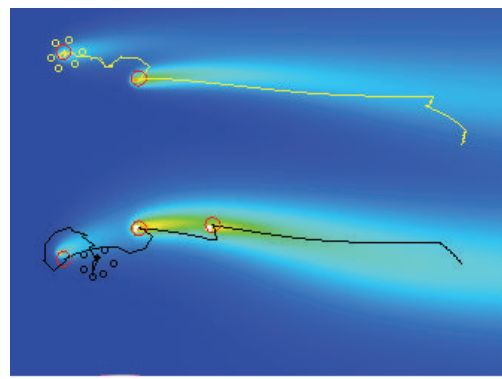

(a) $r_{v T}=0.3 \mathrm{~m}$

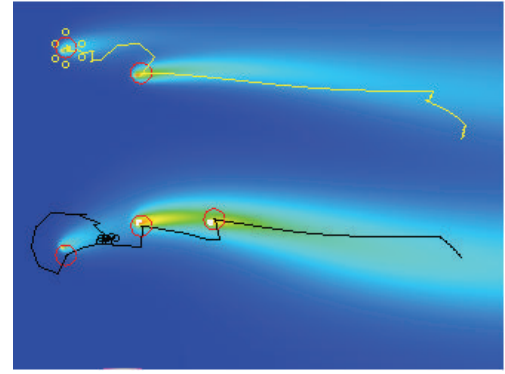

(b) $r_{v T}=0.4 \mathrm{~m}$

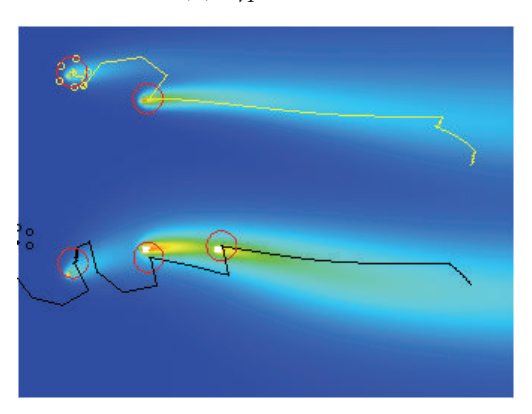

(d) $r_{v T}=0.6 \mathrm{~m}$

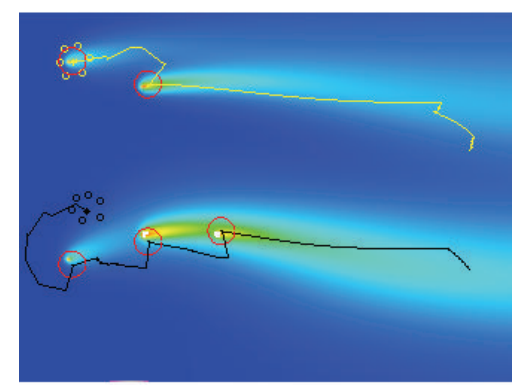

(c) $r_{v T}=0.5 \mathrm{~m}$

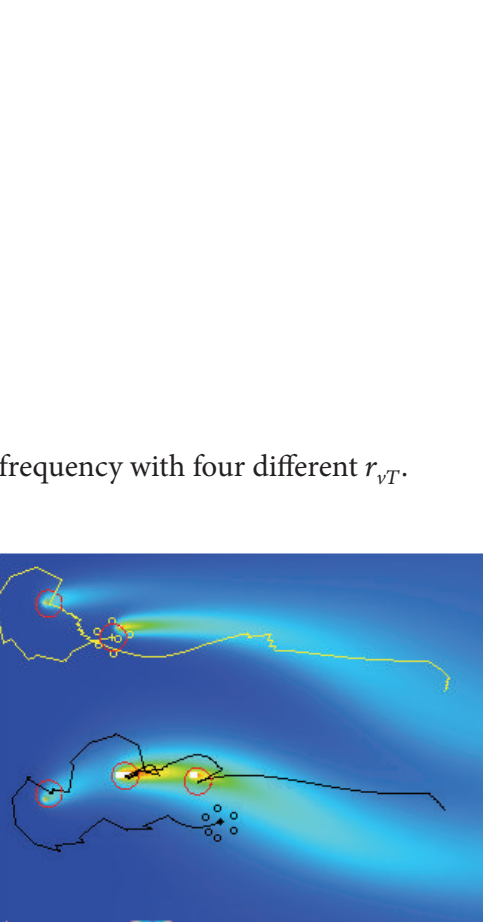

(a) $r_{v T}=0.3 \mathrm{~m}$

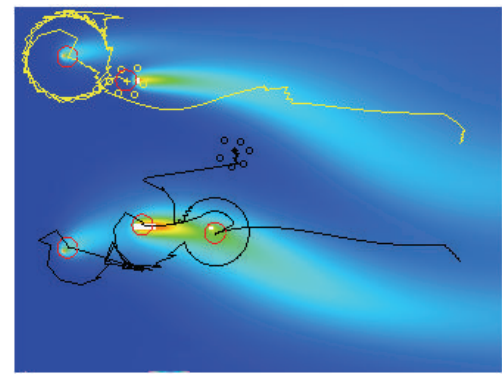

(b) $r_{v T}=0.4 \mathrm{~m}$

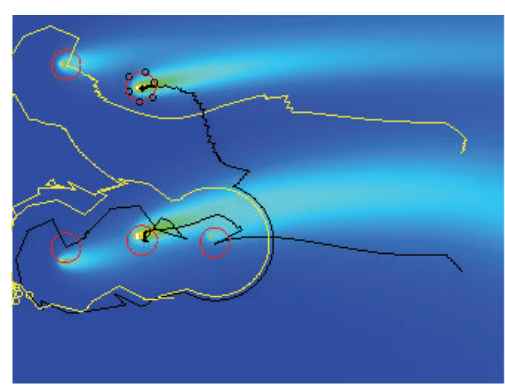

(d) $r_{v T}=0.6 \mathrm{~m}$ (c) $r_{v T}=0.5 \mathrm{~m}$

FIGURE 15: Paths of chemical source localization using anemotaxis at twice frequency with four different $r_{v T}$.

of moving out of the search area, we choose $r_{v T}=0.6 \mathrm{~m}$ for one group search.

\subsection{Performance Comparison on Different Plume Tracing} Algorithm. Two measures are adopted to evaluate the performance of an algorithm in this section: the number of the chemical sources that the robots successfully locate and the time consumption of localizing every chemical source by the robots.

5.2.1. Simulation Results in Case 3 Using Parallel Search by Two Groups. We choose 0.4 for forbidden area radius and the other parameter as same as Table 4 . Since, the plum tracing paths using anemotaxis at three different frequencies have 


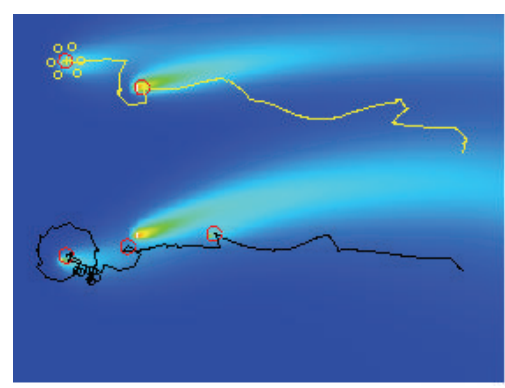

(a) $r_{v T}=0.3 \mathrm{~m}$

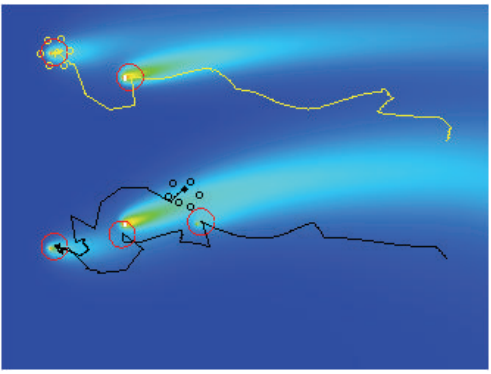

(b) $r_{v T}=0.4 \mathrm{~m}$

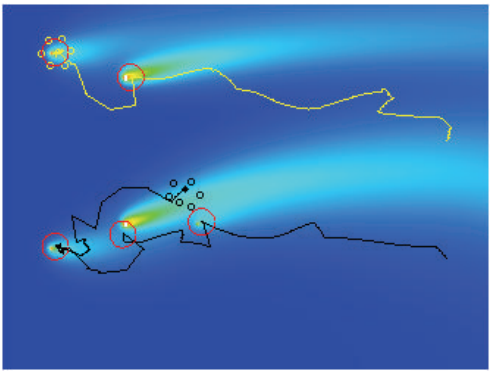

(c) $r_{v T}=0.5 \mathrm{~m}$

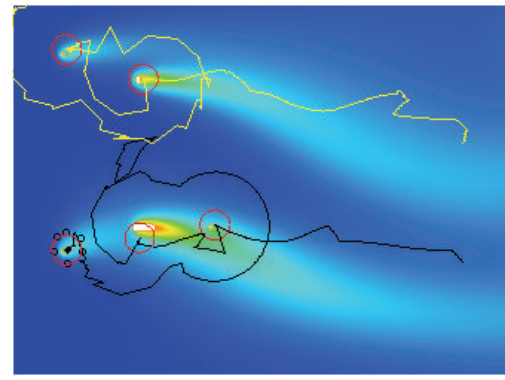

(d) $r_{v T}=0.6 \mathrm{~m}$

FIGURE 16: Paths of chemical source localization using anemotaxis at treble frequency with four different $r_{v T}$.

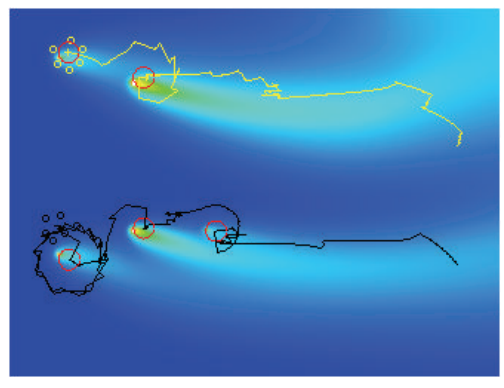

(a) Standard frequency

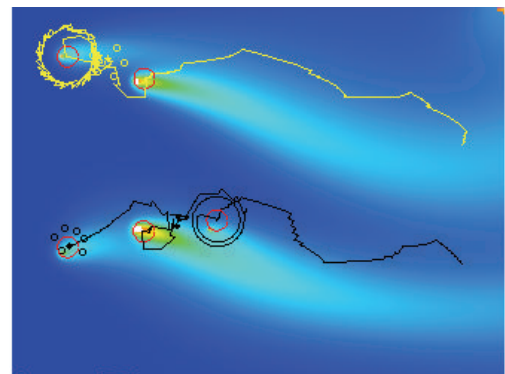

(b) Twice frequency

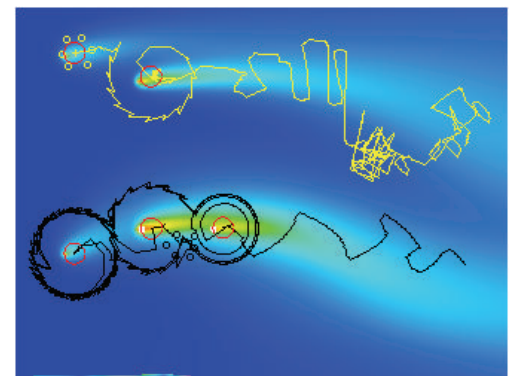

(c) Treble frequency

FIGURE 17: Plume tracing paths using chemotaxis at three different frequencies.

been presented in Figures 14(b)-16(b), we only give the plum tracing paths using chemotaxis at three different frequencies in Figure 17. Table 6 shows the performance comparison of chemotaxis and anemotaxis at three different frequencies.

Figure 17 tells us that chemotaxis is effective and obtains $100 \%$ success rate. However, at third frequency, the tracing paths of the robots indicated by yellow " $\bigcirc$ " are very twist only after they just start. The reason is that, at this region, the plumes from the up and down sources combined together. When the strand of plume from up sources flows to this region, the robots using chemotaxis move upward. While, when the strand of plume from down sources flows to this region, the robots using chemotaxis move downward. This would explain that, for some complex plume, chemotaxis has poor performance. From Table 6, we can see that, with the increasing of the wind direction/speed frequency and methane release frequency, the tracing paths of the robots using anemotaxis is very similar to each other, so the search time used by the robots is also similar. The main reason is that the increasing of the wind direction/speed frequency and methane release frequency make the plume to high concentrations which always exceeded threshold $\rho_{T}$ and the robots using the anemotaxis move towards the upwind direction which make the two groups run and find chemical sources separately and make the search time increase slightly. So, anemotaxis is more effective in parallel search than chemotaxis. For example, in order to find all five sources, the average time consumption used by two groups of robots using anemotaxis is $432 \mathrm{~s}$; while the average time consumption is 956 s using chemotaxis.

5.2.2. Simulation Results in Case 3 Using One Group of Robots. Then, we use one group of robots start from $(18,5)$ optimizing Case 3. We choose 0.6 for forbidden area radius and the other parameter as same as Table 4. Figures 18 and 19 give the plume tracing paths using chemotaxis and anemotaxis at 
TABLE 6: Comparisons on search performance at different frequencies.

\begin{tabular}{|c|c|c|c|c|c|c|}
\hline & \multicolumn{3}{|c|}{ Chemotaxis } & \multicolumn{3}{|c|}{ Anemotaxis } \\
\hline & $\begin{array}{l}\text { Standard } \\
\text { frequency }\end{array}$ & $\begin{array}{c}\text { Twice } \\
\text { frequency }\end{array}$ & $\begin{array}{c}\text { Treble } \\
\text { frequency }\end{array}$ & $\begin{array}{l}\text { Standard } \\
\text { frequency }\end{array}$ & $\begin{array}{c}\text { Twice } \\
\text { frequency }\end{array}$ & $\begin{array}{c}\text { Treble } \\
\text { frequency }\end{array}$ \\
\hline \multicolumn{7}{|c|}{ Number of sources located } \\
\hline 1 & 225 & 252 & 285 & 174 & 173 & 181 \\
\hline 2 & 281 & 562 & 592 & 230 & 242 & 257 \\
\hline 3 & 331 & 622 & 825 & 254 & 307 & 278 \\
\hline 4 & 562 & 709 & 1461 & 280 & 343 & 284 \\
\hline 5 & 534 & 779 & 1554 & 315 & 658 & 323 \\
\hline Average time & & 956 & & & 432 & \\
\hline
\end{tabular}

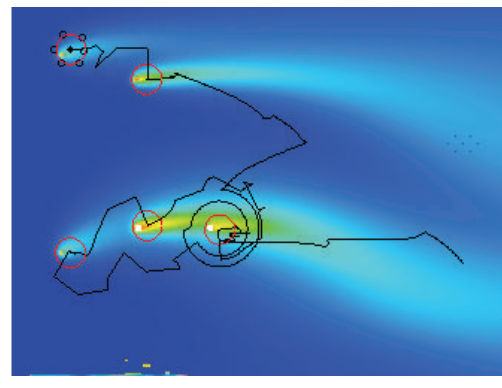

(a) Standard frequency

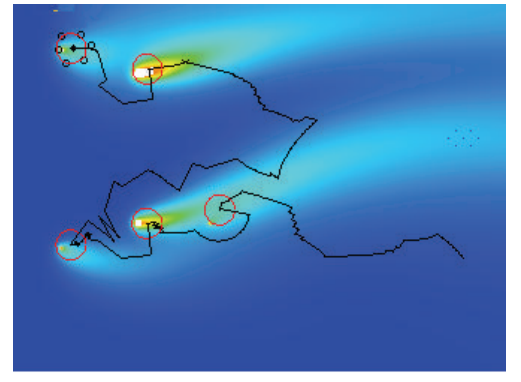

(b) Twice frequency

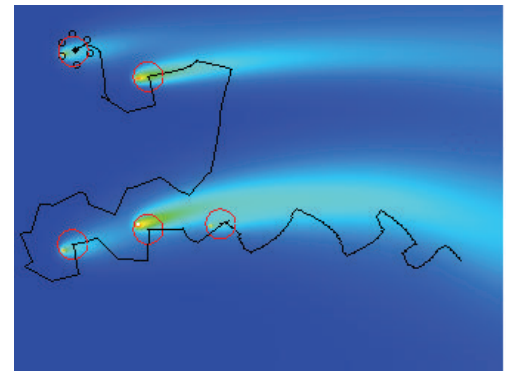

(c) Treble frequency

FIGURE 18: Plume tracing paths using chemotaxis with one group robots at three different frequencies.

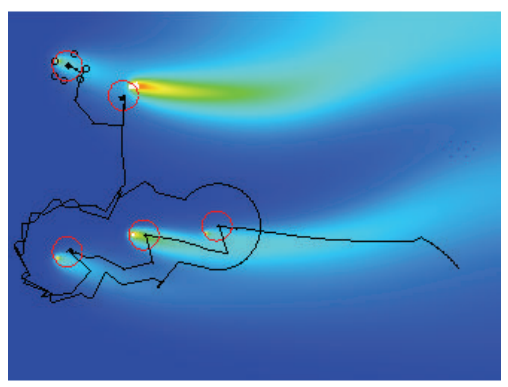

(a) Standard frequency

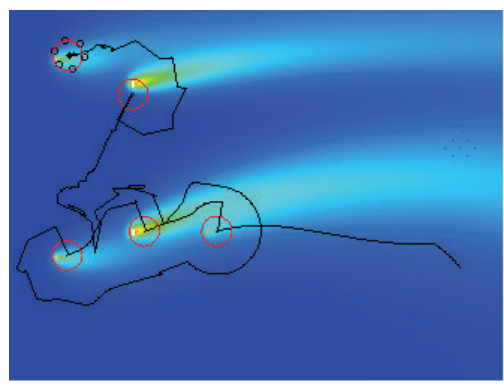

(b) Twice frequency

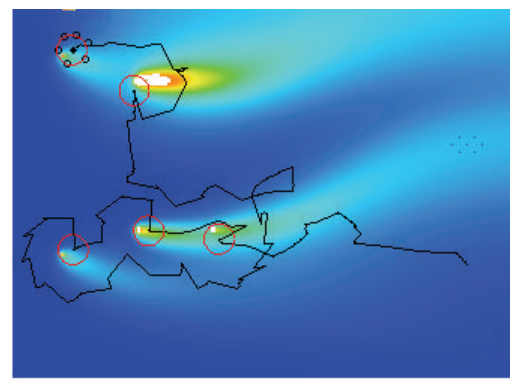

(c) Treble frequency

FIGURE 19: Plume tracing paths using anemotaxis with one group robots at three different frequencies.

three different frequencies, respectively. And Table 7 shows the performance comparison of chemotaxis and anemotaxis at three different frequencies.

Figures 18 and 19 and Table 7 tell us that one group of robots could perform five sources localization in Case 3 . Since the initial position of the robots is in the plume of down three sources Source 1, Source 3 and Source 5, the robots can locate them easily. In order to locate the other two sources Source 2 and Source 4, the robots should move to the plume of up sources. As be seen from Figures 18 and 19, the robots successfully escape from the plume of down sources with the help of three set forbidden area, rotary force and goal force, move to the plume of up sources and locate all five sources. It is worth noting that the goal force which make the robots escape from the down plume is chemotaxis, not anemotaxis. So, we can see from the Table 7 , when using one group of robots chemotaxis is more effective than anemotaxis. We also admit that, when the two strands of plumes are far apart, this strategy is not feasible. In this case, we may increase the self-exploration of the robots or use parallel search by more groups' robots to solve this problem.

Although one group of robots could perform five sources localization in Case 3, the time consumption is much more than parallel search by two groups' robots. For example, using anemotaxis, the average time consumption used by one group of robots is $715 \mathrm{~s}$; while the average time consumption is $432 \mathrm{~s}$ by two groups' robots (see Tables 6 and 7).

\section{Conclusion}

The problem of locating multiple chemical sources is discussed, and a method of simultaneously locating chemical 
TABLE 7: Comparisons on search performance at different frequencies.

\begin{tabular}{cccccc}
\hline & Standard & Chemotaxis & & \multicolumn{2}{c}{ Anemotaxis } \\
frequency & $\begin{array}{c}\text { Twice } \\
\text { frequency }\end{array}$ & $\begin{array}{c}\text { Treble } \\
\text { frequency }\end{array}$ & $\begin{array}{c}\text { Standard } \\
\text { frequency }\end{array}$ & $\begin{array}{c}\text { Treble } \\
\text { frequency }\end{array}$ \\
frequency
\end{tabular}

sources using robots based on virtual physics with release strategy is presented in this study. The release strategy includes setting forbidden area, releasing the robots from declared sources, and escaping from it by a rotary force and goal force. The proposed release strategy can avoid the robots relocating the same source which has been located by other robots and lead them to move toward other sources. Various turbulent plume environments are simulated by Fluent and Gambit software, and the proposed method was employed to locate chemical sources in various scenarios. Simulation experiments compared two plume-tracing algorithms: chemotaxis and anemotaxis and discussed the influence of the varied wind direction/speed frequencies and methane release frequencies and different initial positions of the robots to the search performance. The experimental results show that the proposed strategy is suitable for multiple chemical sources localization even in environment where multiple plumes overlap.

However, this study assumes that the robot can sense the information precisely. In fact, the measurement results have somewhat errors or noises. So how to locate multiple chemical sources in a noisy environment is one topic of our future research. Also, the presented method in the experiments observed that the radius of the forbidden area requires to be defined prior to starting the search; how to define a methodology to adapt this parameter at runtime is another topic of our future research. In addition, this study is performed in simulated environment; so how to verify the performance of the proposed strategy in real-world environments is the third research topic.

\section{Conflict of Interests}

The authors declare that there is no conflict of interests regarding the publication of this paper.

\section{Acknowledgments}

This work was financially supported by the National Natural Science Foundation of China under Grant 61303183, the Natural Science Foundation of Jiangsu province under Grant BK20130204, the Specialized Research Fund for the Doctoral Program of Higher Education of China under Grant
20120095120023, and the Fundamental Research Funds for the Central Universities under Grant 2013XK09.

\section{References}

[1] J. Atema, "Chemical signals in the marine environment: dispersal, detection, and temporal signal analysis," Proceedings of the National Academy of Sciences of the United States of America, vol. 92, no. 1, pp. 62-66, 1995.

[2] Q. Lu, Q.-L. Han, X. Xie, and S. Liu, "A finite-time motion control strategy for odor source localization," IEEE Transactions on Industrial Electronics, vol. 61, no. 10, pp. 5419-5430, 2014.

[3] S. Pang and J. A. Farrell, "Chemical plume source localization," IEEE Transactions on Systems, Man, and Cybernetics, Part B: Cybernetics, vol. 36, no. 5, pp. 1068-1080, 2006.

[4] J. A. Farrell, S. Pang, W. Li, and R. Arrieta, "Chemical plume tracing experimental results with a REMUS AUV," in Proceedings of the OCEANS, vol. 2, pp. 962-968, San Diego, Calif, USA, September 2003.

[5] M. Vergassola, E. Villermaux, and B. I. Shraiman, "'Infotaxis' as a strategy for searching without gradients," Nature, vol. 445, no. 7126, pp. 406-409, 2007.

[6] G. Ferri, E. Caselli, V. Mattoli, A. Mondini, B. Mazzolai, and P. Dario, "SPIRAL: a novel biologically-inspired algorithm for gas/odor source localization in an indoor environment with no strong airflow," Robotics and Autonomous Systems, vol. 57, no. 4, pp. 393-402, 2009.

[7] J. H. Belanger and M. A. Willis, "Biologically-inspired search algorithms for locating unseen odor sources," in Proceedings of the IEEE International Symposium on Intelligent Control Held Jointly with the IEEE International Symposium on Computational Intelligence in Robotics and Automation, and the IEEE International Symposium on Intelligent Systems and Semiotics (ISIC-CIRA-ISAS '98), pp. 265-270, Gaithersburg, Md, USA, September 1998.

[8] Y. Kuwana and I. Shimoyama, "A pheromone-guided mobile robot that behaves like a silkworm moth with living antennae as pheromone sensors," The International Journal of Robotics Research, vol. 17, no. 9, pp. 924-933, 1998.

[9] L. L. López, V. Vouloutsi, A. E. Chimeno et al., "Moth-like chemo-source localization and classification on an indoor autonomous robot," in On Biomimetics, chapter 21, pp. 453-466, InTech, 2011.

[10] R. A. Russell, A. Bab-Hadiashar, R. L. Shepherd, and G. G. Wallace, "A comparison of reactive robot chemotaxis algorithms," Robotics and Autonomous Systems, vol. 45, no. 2, pp. 83-97, 2003. 
[11] W. Jatmiko, K. Sekiyama, and T. Fukuda, "A PSO-based mobile robot for odor source localization in dynamic advectiondiffusion with obstacles environment: theory, simulation and measurement," IEEE Computational Intelligence Magazine, vol. 2, no. 2, pp. 37-51, 2007.

[12] J. Zhang, D. Gong, and Y. Zhang, "A niching PSO-based multi-robot cooperation method for localizing odor sources," Neurocomputing, vol. 123, pp. 308-317, 2014.

[13] K. N. Krishnanand and D. Ghose, "Theoretical foundations for rendezvous of glowworm-inspired agent swarms at multiple locations," Robotics and Autonomous Systems, vol. 56, no. 7, pp. 549-569, 2008.

[14] G. C. H. E. de Croon, L. M. O'Connor, C. Nicol, and D. Izzo, "Evolutionary robotics approach to odor source localization," Neurocomputing, vol. 121, pp. 481-497, 2013.

[15] G. Kowadlo and R. A. Russell, "Using naïve physics for odor localization in a cluttered indoor environment," Autonomous Robots, vol. 20, no. 3, pp. 215-230, 2006.

[16] H. Ishida, H. Tanaka, H. Taniguchi, and T. Moriizumi, "Mobile robot navigation using vision and olfaction to search for a gas/odor source," Autonomous Robots, vol. 20, no. 3, pp. 231238, 2006.

[17] A. Marjovi and L. Marques, "Multi-robot olfactory search in structured environments," Robotics and Autonomous Systems, vol. 59, no. 11, pp. 867-881, 2011.

[18] A. Marjovi, J. G. Nunes, L. Marques, and A. de Almeida, "Multi-robot exploration and fire searching," in Proceedings of the IEEE/RSJ International Conference on Intelligent Robots and Systems (IROS '09), pp. 1929-1934, IEEE, October 2009.

[19] W. Burgard, M. Moors, C. Stachniss, and F. E. Schneider, "Coordinated multi-robot exploration," IEEE Transactions on Robotics, vol. 21, no. 3, pp. 376-386, 2005.

[20] F. Tang and L. E. Parker, "A complete methodology for generating multi-robot task solutions using ASyMTRe-D and marketbased task allocation," in Proceedings of the IEEE International Conference on Robotics and Automation (ICRA '07), pp. 33513358, Roma, Italy, April 2007.

[21] W. M. Spears, D. F. Spears, J. C. Hamann, and R. Heil, "Distributed, physics-based control of swarms of vehicles," Autonomous Robots, vol. 17, no. 2-3, pp. 137-162, 2004.

[22] D. Zarzhitsky, D. F. Spears, and W. M. Spears, "Distributed robotics approach to chemical plume tracing," in Proceedings of the IRS/RSJ International Conference on Intelligent Robots and Systems (IROS '05), pp. 4034-4039, August 2005.

[23] Y. L. Zhang, X. P. Ma, and Y. Z. Miao, "Strategies for locating multiple odor sources using glowworm swarm optimization," TELKOMNIKA Indonesian Journal of Electrical Engineering, vol. 11, no. 7, pp. 3718-3727, 2013.

[24] Y. Zhang, X. Ma, and Y. Miao, "Localization of multiple odor sources using modified glowworm swarm optimization with collective robots," in Proceedings of the 30th Chinese Control Conference (CCC '11), pp. 1899-1904, Yantai, China, July 2011.

[25] D. H. Luo, Y. H. Zou, and J. J. Zhuang, "Multi-robot odor source localization strategy based on a modified ant colony algorithm," Robot, vol. 30, no. 6, pp. 536-541, 2008.

[26] W. Jatmiko, A. Nugraha, R. Effendi et al., "Localizing multiple odor Sources in a dynamic environment based on modified niche particle swarm optimization with flow of wind," WSEAS Transactions on Systems, vol. 8, no. 11, pp. 1187-1196, 2009.

[27] Y. L. Zhang, X. P. Ma, and Y. Z. Miao, "Chemical source localization using mobile robots in indoor arena," TELKOMNIKA
Indonesian Journal of Electrical Engineering, vol. 11, no. 7, pp. 3718-3727, 2013.

[28] X. Ma, Y. Zhang, and Y. Miao, "A virtual physics-based approach to multiple odor sources localization," TELKOMNIKA Indonesian Journal of Electrical Engineering, vol. 12, no. 7, pp. 5331-5341, 2014.

[29] Z. Liu and T. F. Lu, "A simulation framework for plumetracing research," in Proceedings of the Australian Conference on Robotics \& Automation, pp. 1-7, Canberra, Australia, 2008.

[30] T. F. Lu, "Indoor odour source localisation using robot: initial location and surge distance matter?" Robotics and Autonomous Systems, vol. 61, no. 6, pp. 637-647, 2013.

[31] H. Rezaee and F. Abdollahi, "Mobile robots cooperative control and obstacle avoidance using potential field," in Proceedings of the IEEE/ASME International Conference on Advanced Intelligent Mechatronics (AIM '11), pp. 61-66, July 2011. 


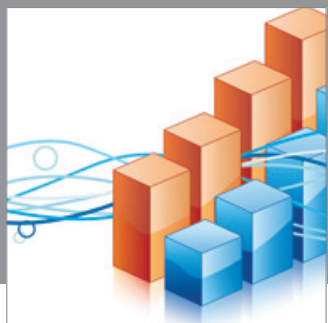

Advances in

Operations Research

mansans

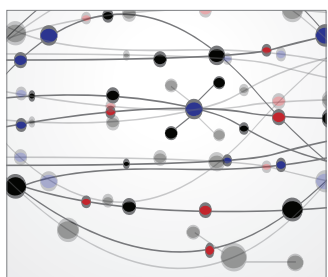

The Scientific World Journal
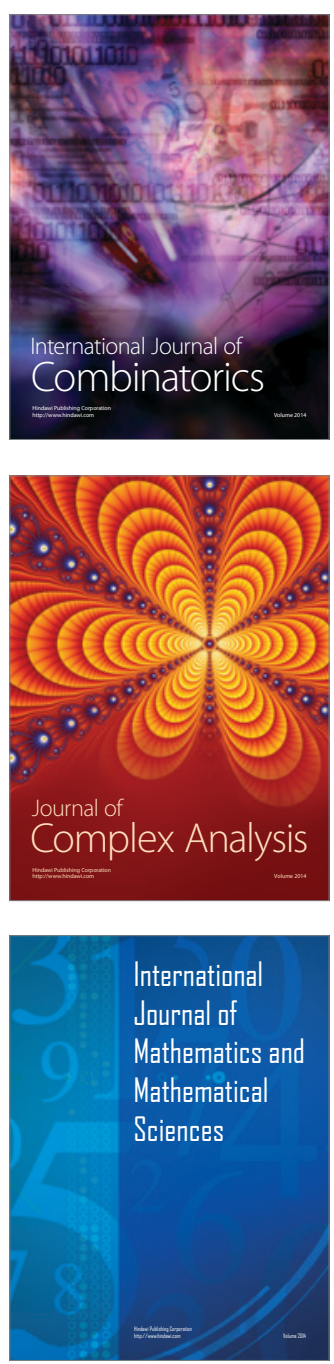
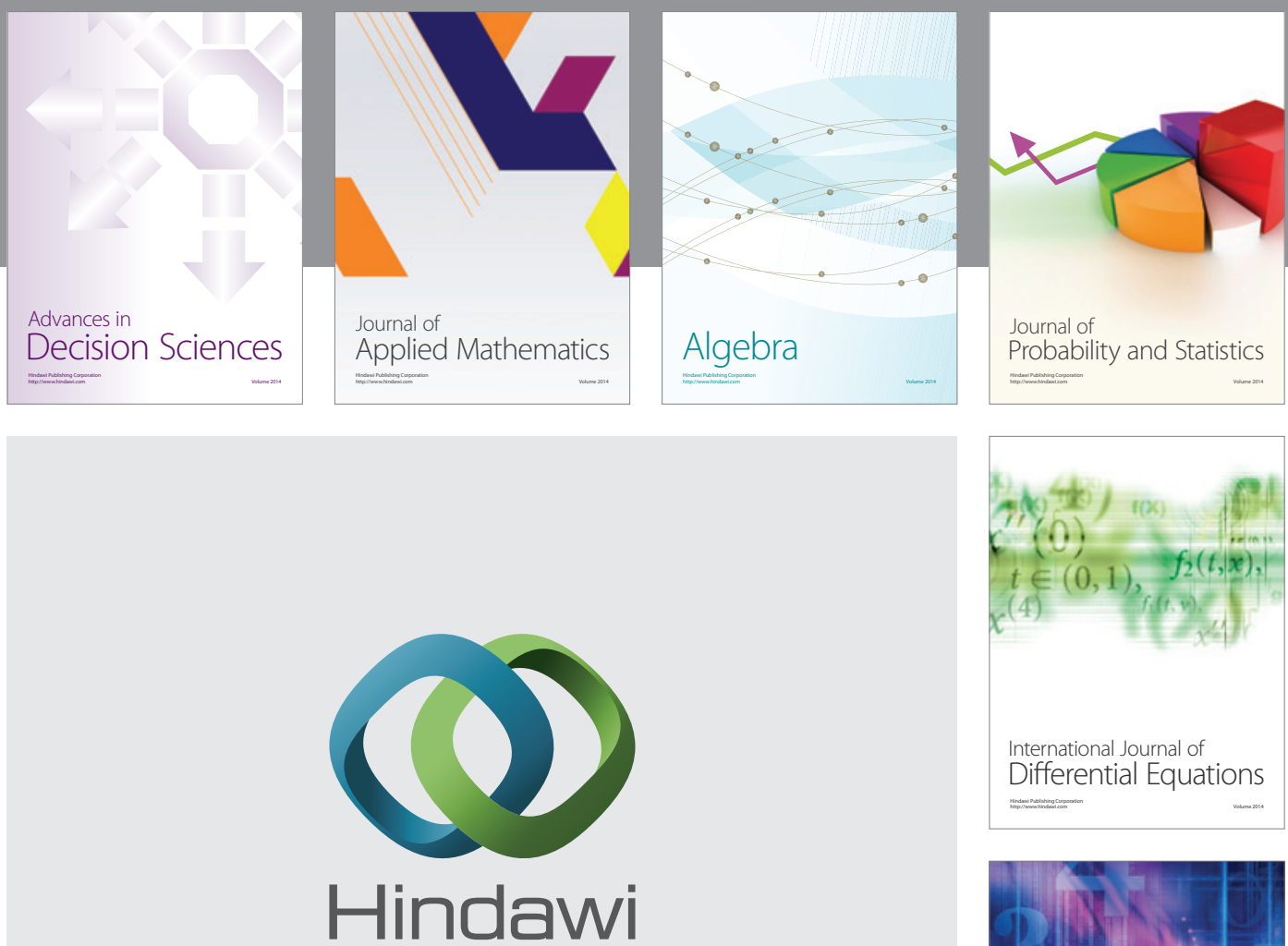

Submit your manuscripts at http://www.hindawi.com
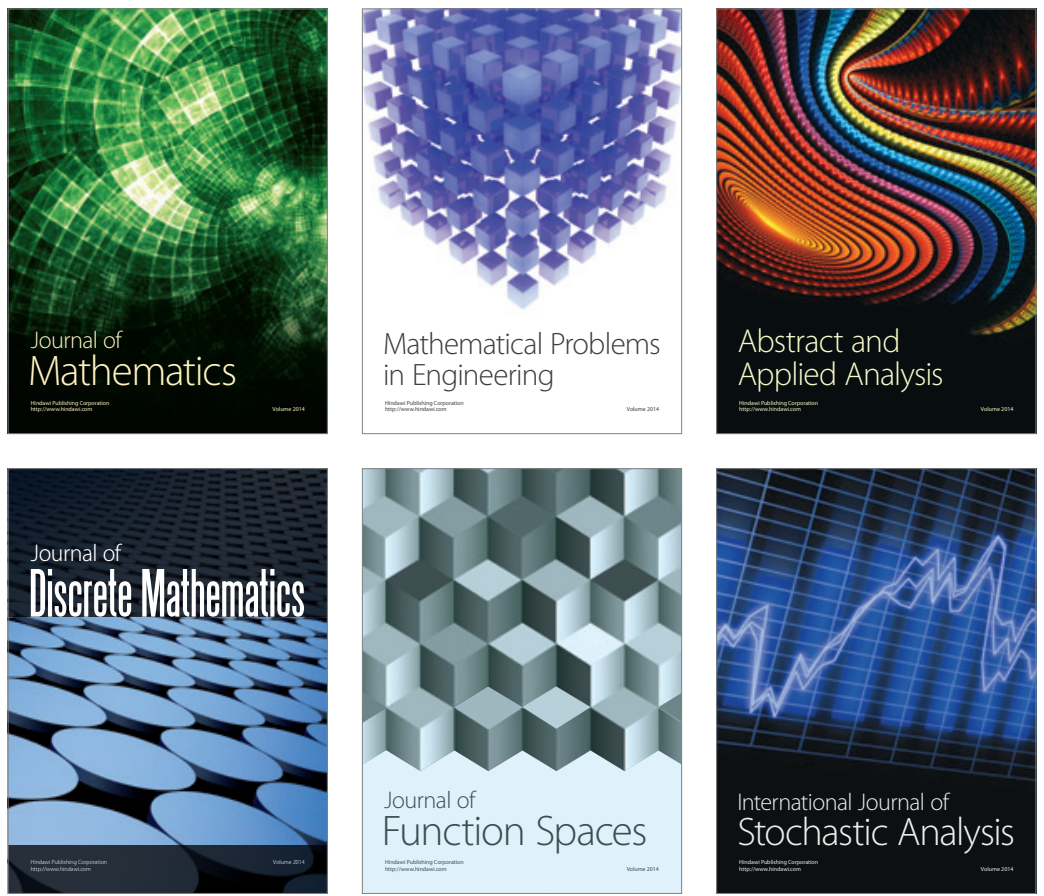

Journal of

Function Spaces

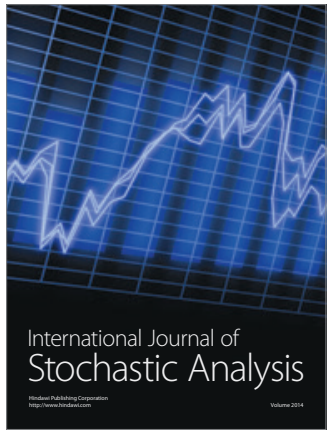

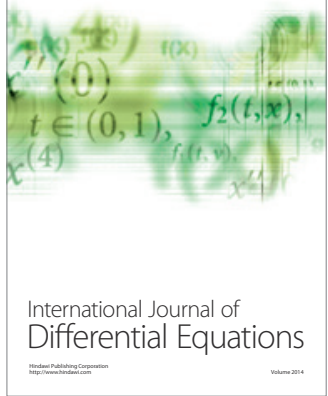
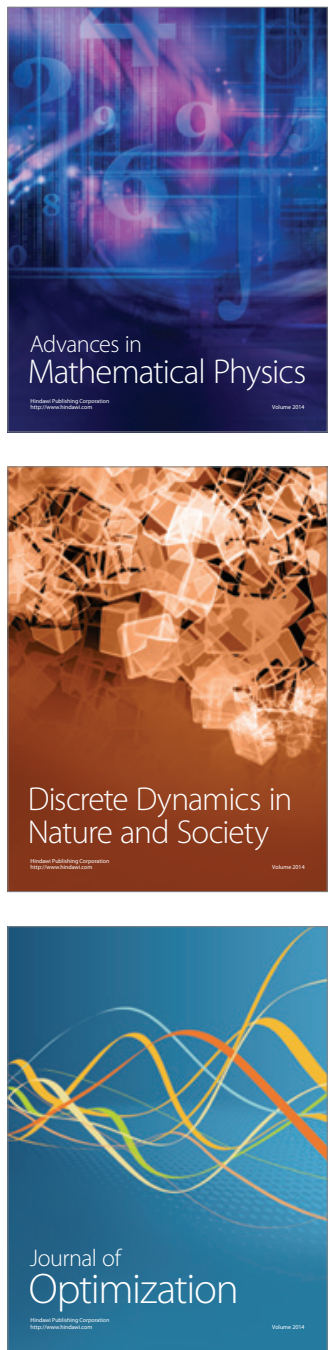